\title{
Fbxw7 regulates lipid metabolism and cell fate decisions in the mouse liver
}

\author{
Ichiro Onoyama, ${ }^{1,2}$ Atsushi Suzuki, 3,4 Akinobu Matsumoto,, ${ }^{1,2}$ Kengo Tomita, ${ }^{5}$ Hideki Katagiri, ${ }^{6}$ \\ Yuichi Oike, ${ }^{4,7}$ Keiko Nakayama, ${ }^{2,8}$ and Keiichi I. Nakayama ${ }^{1,2}$
}

\begin{abstract}
${ }^{1}$ Department of Molecular and Cellular Biology, Medical Institute of Bioregulation, Kyushu University, Higashi-ku, Fukuoka, Fukuoka, Japan. ${ }^{2}$ CREST, Japan Science and Technology Agency, Kawaguchi, Saitama, Japan. ${ }^{3}$ Division of Organogenesis and Regeneration, Medical Institute of Bioregulation,

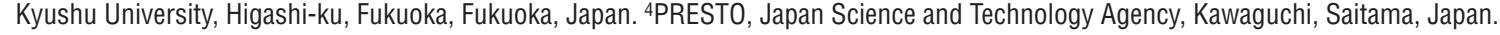
${ }^{5}$ Division of Gastroenterology and Hepatology, Department of Internal Medicine, National Defense Medical College, Tokorozawa, Saitama, Japan. ${ }^{6}$ Division of Advanced Therapeutics for Metabolic Diseases, Center for Translational and Advanced Animal Research,

Tohoku University Graduate School of Medicine, Sendai, Japan. ${ }^{7}$ Department of Molecular Genetics, Graduate School of Medical Sciences, Kumamoto University, Kumamoto, Japan. ${ }^{8}$ Division of Developmental Genetics, Center for Translational and Advanced Animal Research, Tohoku University Graduate School of Medicine, Sendai, Japan.
\end{abstract}

\begin{abstract}
E3 ubiquitin ligase complexes of the SCF type consist of ring-box 1 (Rbx1), cullin 1 (Cul1), S-phase kinase-associated protein 1 (Skp1), and a member of the F-box family of proteins. The identity of the F-box protein determines the substrate specificity of the complex. The F-box family member F-box-and WD repeat domain-containing 7 (Fbxw7; also known as Fbw7, SEL-10, hCdc4, and hAgo) targets for degradation proteins with wide-ranging functions, and uncovering its in vivo role has been difficult, because $F b x w 7^{-/-}$embryos die in utero. Using two different Cre-loxP systems ( $M x 1$-Cre and $A l b$-Cre), we generated mice with liver-specific null mutations of Fbxw 7 . Hepatic ablation of $F b x w 7$ resulted in hepatomegaly and steatohepatitis, with massive deposition of triglyceride, a phenotype similar to that observed in humans with nonalcoholic steatohepatitis. Both cell proliferation and the abundance of $\mathrm{Fbxw} 7$ substrates were increased in the Fbxw7-deficient liver. Long-term Fbxw7 deficiency resulted in marked proliferation of the biliary system and the development of hamartomas. Fbxw7 deficiency also skewed the differentiation of liver stem cells toward the cholangiocyte lineage rather than the hepatocyte lineage in vitro. This bias was corrected by additional loss of the Notch cofactor RBP-J, suggesting that Notch accumulation triggered the abnormal proliferation of the biliary system. Together, our results suggest that Fbxw7 plays key roles, regulating lipogenesis and cell proliferation and differentiation in the liver.
\end{abstract}

\section{Introduction}

The abundance of cellular proteins is regulated in a coordinated manner at the levels of their synthesis and degradation. In particular, intracellular proteolysis is thought to be subject to highly specific regulation. The ubiquitin-proteasome system is responsible for such specific degradation of proteins, with ubiquitylation playing the regulatory role in this process. Ubiquitylation of target proteins is mediated by the sequential action of 3 enzymes: a ubiquitin-activating enzyme (E1), a ubiquitin-conjugating enzyme (E2), and a ubiquitin ligase (E3). The ubiquitylated substrates are then selectively recognized and degraded by the $26 \mathrm{~S}$ proteasome (1). Uncontrolled proteolysis is implicated in dysregulation of cell proliferation and aberrant cell differentiation and is thought to underlie many human malignancies (2).

F-box proteins determine the substrate specificity of the SCF-type E3 complex, which consists of the RING-finger protein ring-box 1 (Rbx1; also known as Roc1 and Hrt1), the scaffold protein cullin 1 (Cul1), and the adaptor protein S-phase kinase-associated protein 1 (Skp1) in addition to an F-box protein (2-4). F-box- and WD repeat domain-containing 7 (Fbxw7; also known as Fbw7, SEL-10, hCdc4, and hAgo) is a member of the F-box protein family that was initially identified as a negative regulator of LIN-12-mediated (Notchmediated) signaling in Caenorhabditis elegans by genetic analysis (5, 6). Fbxw7 also interacts with Notch family proteins and promotes their ubiquitin-dependent turnover in mammalian cells $(5,7,8)$.

Conflict of interest: The authors have declared that no conflict of interest exists. Citation for this article: J Clin Invest. 2010;121(1):342-354. doi:10.1172/JCI40725.
Furthermore, it targets for degradation various mammalian proteins that control cell cycle progression $(2,4)$, including cyclin $\mathrm{E}$ (9-11), c-Myc (12,13), and c-Jun $(14,15)$, as well as other proteins that do not contribute directly to cell cycle control, such as SREBPs (16-18), mammalian target of rapamycin (mTOR) (19), and PPAR- $\gamma$ coactivator-1 $\alpha$ (PGC-1 $\alpha)(20)$.

Given its ability to promote degradation of cyclin E, c-Myc, c-Jun, and Notch, all of which are products of proto-oncogenes, Fbxw7 was expected to function as an oncosuppressor protein. Indeed, mutations in the $F b x w 7$ gene have been detected in many types of human malignancy, including cholangiocarcinoma and $\mathrm{T}$ cell acute lymphoblastic leukemia as well as pancreatic, gastric, colorectal, prostate, and endometrial cancer (21-31). The study of Fbxw7 is thus important not only from the point of view of basic biology but also from the medical standpoint.

To analyze the functions of $\mathrm{Fbxw} 7$ in vivo, we and others have generated Fbxw7-deficient mice. However, Fbxw $7-/$ embryos were found to die in utero at E10.5, manifesting marked abnormalities in vascular development as a result of dysregulation of Notch signaling $(32,33)$. To avoid this early embryonic mortality, we have established mice in which $F b x w 7$ is conditionally disrupted in T cells (34) or in hematopoietic stem cells (35), and we have also examined the effects of $F b x w 7$ ablation in mouse embryonic fibroblasts (36). The loss of Fbxw7 in immature T cells results in the failure of these cells to exit the cell cycle, leading to thymic hyperplasia and the subsequent development of lymphoma. Among known targets of Fbxw7, only c-Myc and Notch accumulated in the Fbxw7-deficient thymocytes, and c-Myc accumulation was found 
to be primarily responsible for the hyperproliferation phenotype. In contrast to that in immature T cells, the accumulation of c-Myc apparent in Fbxw7-null mature T cells induced expression of p53, which in turn led to cell cycle arrest and apoptosis. Furthermore, we found that Fbxw7 contributes to the long-term maintenance of hematopoietic stem cells. Most of the phenotypes of Fbxw7 deficiency in these conditional mouse mutants are related to cell proliferation or death and appear to be attributable to deregulation of c-Myc and Notch. Although Fbxw7 targets many substrates that do not participate directly in cell cycle control for degradation, the physiological roles of Fbxw7-mediated degradation of such targets have been largely unclear.

We have now examined the consequences of Fbxw7 deficiency in the liver. Unexpectedly, the major phenotypes associated with such deficiency were abnormalities in lipid metabolism and cell differentiation, which differ markedly from those in hematopoietic cell lineages and fibroblasts, in which Fbxw7 contributes primarily to the control of cell proliferation and apoptosis. We thus propose that Fbxw7 targets different groups of proteins for ubiquitin-dependent degradation and thereby contributes to distinct biological functions in a tissue-specific manner.

\section{Results}

Conditional inactivation of Fbxw 7 in the liver by 2 Cre-loxP systems. We generated mice harboring floxed $F b x w 7$ alleles (referred to herein as $F b x w 7^{F / F}$ mice) in which exon 5 (which encodes the F-box domain) is flanked by loxP sites (34). To ablate Fbxw 7 in the liver, we crossed these $F b x w 7^{F / F}$ mice with mice harboring a Cre transgene under the control of the promoter for the myxovirus resistance 1 (Mx1) or albumin $(A l b)$ genes (Mx1-Cre or Alb-Cre mice). We confirmed that almost all floxed alleles were inactivated by Cre recombinase in the livers of $A l b$-Cre/Fbxw $7^{F / F}$ mice as well as in those of $M x 1$ $\mathrm{Cre} / \mathrm{Fb} x w 7^{F / F}$ mice at 3 weeks after the last of 3 i.p. injections of poly $(\mathrm{I})$-poly $(\mathrm{C})(\mathrm{pI} \mathrm{PC})$ to activate the $M x 1$ gene promoter (Figure 1A). For subsequent experiments, we examined the effects of shortor long-term Fbxw7 deficiency in Mx1-Cre/Fbxw $7^{F / F}$ mice and those of long-term a priori deficiency in $A l b$-Cre/Fbxw $7^{F / F}$ mice.

Massive lipid deposition and nonalcoholic steatohepatitis-like lesions in the Fbxw7-deficient liver. Mx1-Cre/Fbxw $7^{F / F}$ mice at 8 weeks of age were subjected to i.p. injection of $\mathrm{pIpC}$ every other day for 3 days to activate the $M x 1$ gene promoter. At 3 weeks after the last injection of $\mathrm{pIpC}$, the livers of these mice were enlarged and lighter in color compared with those of control animals (Figure 1B). The liver-tobody weight ratio of these $M x 1$-Cre/Fbxw $7^{F / F}$ mice was increased by approximately $30 \%$ relative to that of control mice (Figure $1 \mathrm{C}$ ). Histological examination revealed that the nuclei of cells in the enlarged liver remained centrally located, whereas the corresponding cytoplasm was only weakly eosinophilic and contained numerous microvesicular vacuoles (Figure 1, D and E). Staining with Oil red $\mathrm{O}$ (Figure 1, F-I) also revealed massive lipid deposition, predominantly in the area around central veins (Figure $1 \mathrm{G}$ ). Similar lipid deposition was also observed in the livers of $A l b$-Cre/Fbxw $7^{F / F}$ mice at as early as 12 weeks of age (Figure 1, J and K). The mechanism underlying such an uneven localization of lipid deposition is unclear and awaits further investigation.

Lobular infiltration of inflammatory cells such as lymphocytes and neutrophils (Figure 1, L and M; arrowhead), as well as the presence of many ballooned hepatocytes (occasionally containing Mallory body-like eosinophilic inclusions) (Figure 1, N and O; arrow), were observed in the livers of older mutant mice at approximately
50 weeks of age. Sinusoidal fibrogenic changes in the liver as revealed by Masson's trichrome staining were also evident (Figure 1, $\mathrm{P}$ and Q), and serum levels of aspartate aminotransferase (AST) and alanine aminotransferase (ALT) were significantly increased (Figure 1R) in $A l b$-Cre $/ F b x w 7^{F / F}$ mice at 50 weeks of age. The serum level of bilirubin tended to be higher in the mutant animals than in age-matched controls, suggestive of the destruction of liver tissue in the mutant mice, although this difference was not statistically significant (Supplemental Figure 1; supplemental material available online with this article; doi:10.1172/JCI40725DS1). The onset of inflammatory changes occurred later than that of steatosis, but feeding $M x 1$-Cre/Fbxw $7^{F / F}$ mice a methionine- and choline-deficient (MCD) diet resulted in acceleration of inflammation (Figure $2 \mathrm{~A})$. The extents of steatosis and hepatitis were less pronounced in $A l b$-Cre $/ F b x w 7^{F / F}$ mice than in $M x 1$-Cre $/ F b x w 7^{F / F}$ mice subjected to acute ablation of Fbxw7, probably as a result of compensatory mechanisms operative during development in the former animals. However, massive steatosis and inflammation were also apparent in Alb-Cre/Fbxw $7^{F / F}$ mice fed the MCD diet, whereas control animals did not show such marked changes (Figure 2B). These results suggested that $A l b$-Cre $/ F b x w 7^{F / F}$ mice are also more sensitive to steatohepatitis than are controls. The histological findings in both types of Fbxw7-deficient mice are highly similar to those associated with nonalcoholic steatohepatitis (NASH) in humans (37).

Expression of adipogenic and lipogenic genes in the Fbxw7-deficient liver. We next determined lipid concentrations in liver extracts. Triglyceride levels were significantly increased in the livers of $M x 1$ Cre/Fbxw $7^{F / F}$ mice compared with those in control animals at 3 weeks after the final $\mathrm{pIpC}$ injection, whereas the concentration of total cholesterol was not affected in the mutant livers (Figure 3A). Given that triglyceride synthesis is regulated predominantly by transcriptional activators, such as SREBPs, carbohydrate response element-binding protein (ChREBP), and PPAR- $\gamma$, we examined the expression of these proteins and their downstream targets in the liver. Immunoblot analysis revealed that the abundance of nuclear SREBP1, which is the major SREBP in the liver and a target of Fbxw7-mediated proteolysis $(16,17)$, was increased both in PIpCinjected $M x 1$-Cre/Fbxw $7^{F / F}$ mice and in Alb-Cre/Fbxw $7^{F / F}$ mice (Figure $3 \mathrm{~B}$ and Supplemental Figure 2). The intensity of the more slowly migrating band, likely corresponding to the phosphorylated form of SREBP1, was especially increased, consistent with the previous observation that the phosphorylated forms of SREBPs are targeted by Fbxw7 (16-18), as is generally the case for Fbxw7 substrates (11, 34). In contrast, the amounts of ChREBP and PPAR- $\gamma$ were decreased in the mutant mice compared with those in control animals, suggestive of the operation of a negative feedback loop triggered by triglyceride accumulation. Consistent with this notion, the abundance of Pparg mRNA in the liver was increased in SREBP cleavage-activating protein-deficient mice, in which the SREBP pathway is inactivated (38). The levels of PGC- $1 \alpha$ and mTOR (total or phosphorylated forms) were unaffected by hepatic deletion of Fbxw7.

RT and real-time PCR analysis revealed that the abundance of mRNAs for the adipogenic and lipogenic transcriptional activators SREBP1c, ChREBP, and Pparg was decreased in the Fbxw7-deficient liver (Figure 3C), suggesting that the transcription of these genes is suppressed by a negative feedback loop triggered by the high level of triglyceride. At the protein level, the precursor form of SREBP1 was reduced, probably as a result of the decrease in the abundance of its mRNA, whereas the mature cleaved form was increased (Supplemental Figure 3). Among the downstream targets of SREBPs, 

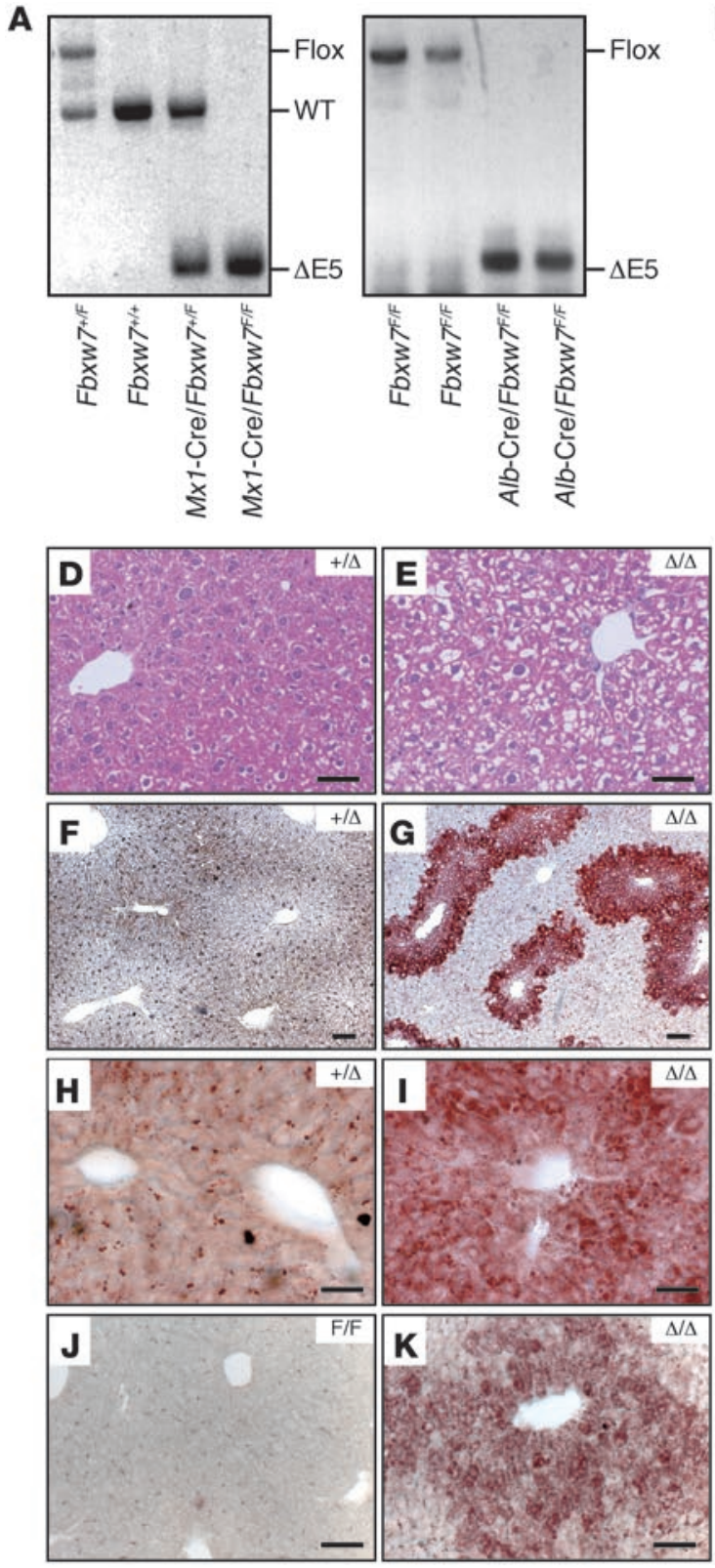

B
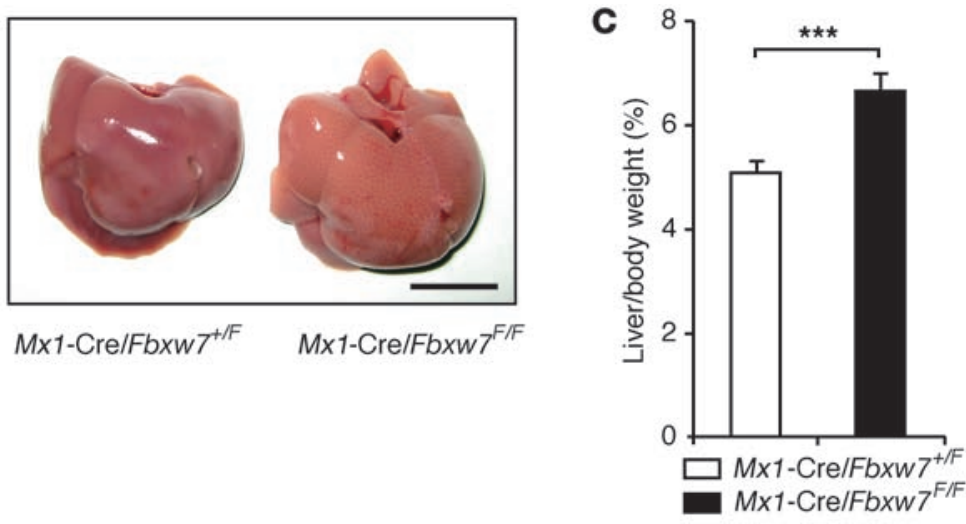
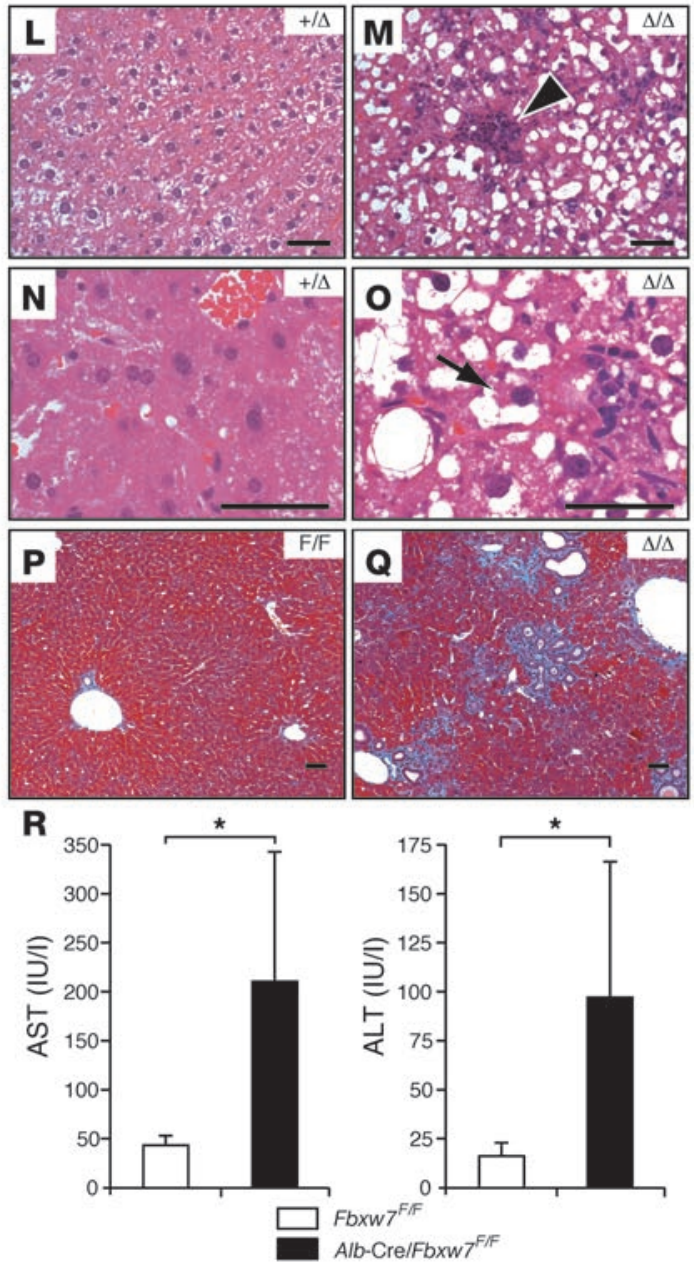

Figure 1

Development of NASH-like liver disease as a result of Fbxw7 deletion. (A) Genomic PCR analysis from the mouse liver of the indicated genotypes. The positions of amplified fragments corresponding to WT, floxed, and exon 5-deleted ( $\Delta \mathrm{E} 5)$ alleles are indicated. (B) Gross appearance of the livers of indicated genotypes treated as in A. Scale bar: $10 \mathrm{~mm}$. (C) Liver/body weight ratio of mice treated as in $\mathbf{A}$. Data are mean \pm SD from 5 animals of each genotype. ${ }^{* * *} P<0.005$. (D and E) H\&E staining of liver sections from $M x 1-\mathrm{Cre} / F b x w 7^{+/ F}(+/ \Delta)$ and $M x 1-\mathrm{Cre} / F b x w 7^{F / F}$ $(\Delta / \Delta)$ mice, respectively, treated as in $\mathbf{A}$. (F and $\mathbf{G})$ Oil red $\mathrm{O}$ staining of liver sections treated as in $\mathbf{A}$. (H and I) Higher-magnification views of images in $\mathbf{F}$ and $\mathbf{G}$, respectively. ( $\mathbf{J}$ and $\mathbf{K})$ Oil red $O$ staining of liver sections from $F b x w 7^{F / F}(\mathrm{~F} / \mathrm{F})$ and $A / b-C r e / F b x w 7^{F / F}(\Delta / \Delta)$ mice, respectively, at 12 weeks of age. (L-O) H\&E staining of liver sections from $M x 1-C r e / F b x w 7^{+/ F}(+/ \Delta)(\mathbf{L}$ and $\mathbf{N})$ and $M x 1-C r e / F b x w 7^{F / F}(\Delta / \Delta)(\mathbf{M}$ and $\mathbf{O}) \mathrm{mice}$ at 50 weeks after the final injection of plpC. Lobular infiltration of inflammatory cells is indicated by the arrowhead, and Mallory body-like eosinophilic inclusion is indicated by the arrow. (P and $\mathbf{Q})$ Masson's trichrome staining of liver sections from $F b x w 7 F / F(F / F)$ and $A / b-C r e / F b x w 7 F / F(\Delta / \Delta)$ mice, respectively, at 50 weeks of age. Scale bar: $50 \mu \mathrm{m}(\mathbf{D}, \mathbf{E}, \mathbf{H}, \mathbf{I}$, and L-O); $100 \mu \mathrm{m}(\mathbf{F}, \mathbf{G}, \mathbf{J}, \mathbf{K}, \mathbf{P}$, and Q). (R) Serum AST and ALT activities in $F b x w 7^{F / F}(n=6)$ and Alb-Cre/Fbxw $7^{F / F}(n=10)$ mice at 50 weeks of age. Data are mean \pm SD. ${ }^{*} P<0.05$. 
A
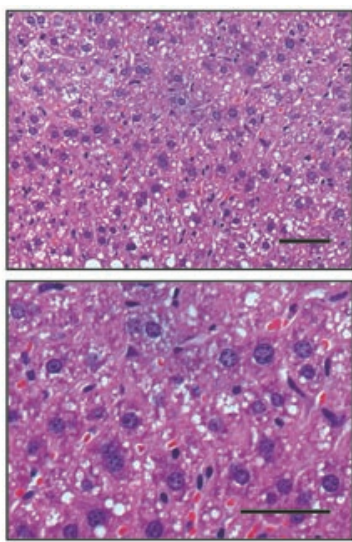

Control

B
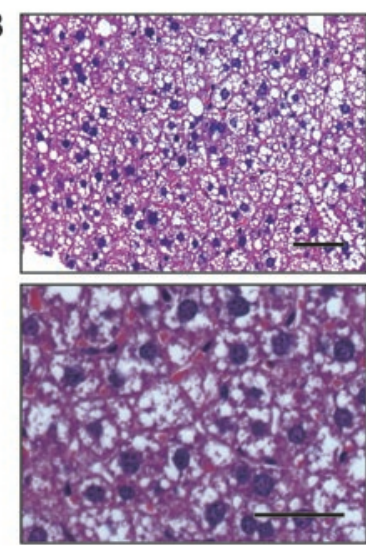

Control
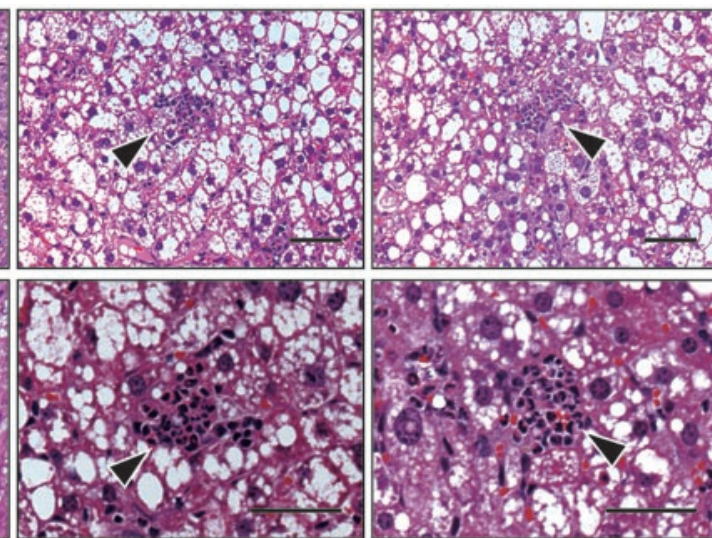

Mx1-Cre/Fbxw7F/F
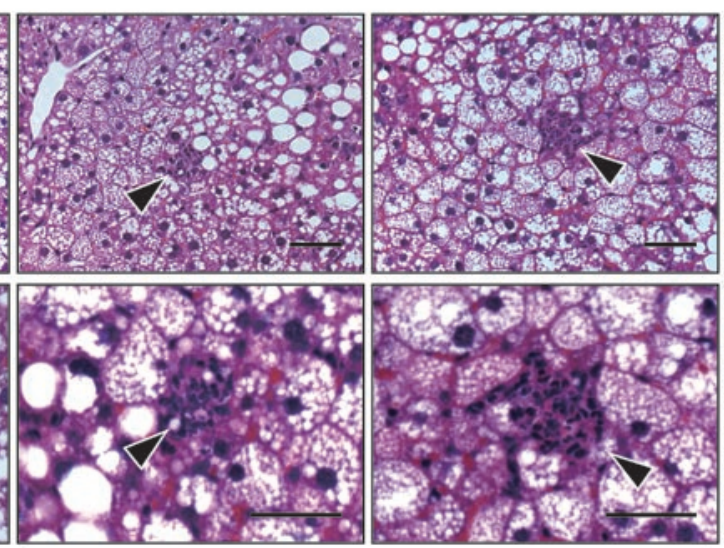

Alb-Cre/Fbxw7F/F

\section{Figure 2}

Increased susceptibility to a NASH-like condition conferred by Fbxw7 ablation in the liver. (A) $M \times 1$ Cre/FbxW $7^{+/ F}$ (control) and Mx1-Cre/FbxW7F/F mice were injected with plpC at 8 weeks of age and then fed an MCD diet for 2 weeks. Liver sections were then subjected to H\&E staining. Lowerand higher-magnification views are shown (top and bottom panels, respectively). In addition to fatty degeneration, many foci of lobular infiltration of inflammatory cells (arrowheads) were apparent in the livers of $M x 1-\mathrm{Cre} / F b x w 7^{F / F}$ mice. Scale bar: $50 \mu \mathrm{m}$. (B) Alb-Cre/Fbxw $7^{+/ F}$ (control) and Alb-Cre/FbxW $7^{F / F}$ mice at 12 weeks of age were fed an MCD diet for 4 weeks and then analyzed as in $\mathbf{A}$. Lower- and higher-magnification views are shown (top and bottom panels, respectively). Control mice developed a small extent of fatty degeneration, whereas Alb-Cre/Fbxw $7^{\mathrm{F} / \mathrm{F}}$ mice showed massive accumulation of lipid droplets and many foci of lobular infiltration of inflammatory cells (arrowheads) similar to those apparent in $M \times 1-C r e / F b x w 7 F / F$ mice. Scale bar: $50 \mu \mathrm{m}$. the amounts of mRNAs for fatty acid synthase (Fas) and stearoylCoA desaturase-1 ( $S c d 1$ ) were increased, whereas those for the LDL receptor $(L d l r)$ and HMG-CoA synthase (Hmgcs1) were decreased, in the mutant liver (Figure $3 \mathrm{C}$ ). Immunostaining also showed that SREBP1 accumulated in the region around the central veins (Figure 3D), corresponding to the area of lipid deposition, even though deletion of Fbxw7 appears to occur throughout almost the entire liver. The expression of SCD-1 was also increased in the region around the central veins in which SREBP1 was upregulated (Figure 3E). Collectively, these results suggested that the accumulation of SREBP proteins as a result of Fbxw7 ablation results in triglyceride deposition in the liver, which in turn affects the expression of other adipogenic and lipogenic genes as well as their downstream targets via a negative feedback loop.

Increased proliferation of Fbxw7-deficient hepatocytes. We compared the abundance of cyclin E and c-Myc between the livers of $M \times 1$ Cre $/ F b x w 7^{+/ F}$ and $M x 1$-Cre $/ F b x w 7^{F / F}$ mice at 3 or 50 weeks after the final $\mathrm{pIpC}$ injection, beginning at 8 weeks of age. Immunoblot analysis revealed that the amount of cyclin $\mathrm{E}$ in the livers of $M \times 1$ $\mathrm{Cre} / \mathrm{Fb} x w 7^{F / F}$ mice was increased compared with that in Mx1-Cre/ $F b x w 7^{+/ F}$ mice at 3 weeks after $\mathrm{pIpC}$ injection but not at 50 weeks (Figure 4A). The abundance of c-Myc was not affected by the loss of Fbxw7 in the liver, at either 3 or 50 weeks after injection. To measure the rate of hepatocyte proliferation, we subjected $M \times 1$ $\mathrm{Cre} / F b x w 7^{+/ F}$ and $M x 1$-Cre $/ F b x w 7^{F / F}$ mice to i.p. injection with $\mathrm{BrdU}$ for 3 consecutive days, beginning at 3 or 50 weeks after the final $\mathrm{pIpC}$ injection. Immunostaining of the liver with antibodies to BrdU at 1 day after the last BrdU injection revealed that the rate of BrdU incorporation was markedly increased in Fbxw7-deficient liver cells compared with that in control cells (Figure 4B). Most of the BrdU-positive cells were also reactive with antibodies to albumin but not with those to cytokeratin 19 (CK19) at 3 weeks after $\mathrm{pIpC}$ injection (Figure $4 \mathrm{C}$ ), suggesting that the proliferating cells are predominantly hepatocytes. In contrast, at 50 weeks after $\mathrm{pIpC}$ injection, both hepatocytes and cholangiocytes in $M x 1$-Cre/Fbxw $7^{F / F}$ mice incorporated BrdU to a greater extent than did those in control mice. The TUNEL assay revealed that the frequency of apoptosis was also increased in the Fbxw7-deficient liver at 3 weeks after $\mathrm{pIpC}$ injection (Figure 4, D and E), suggesting that the loss of Fbxw7 transiently promotes cell cycle progression but eventually results in apoptosis in the liver, as it does in T lymphocytes (34).

Development of hamartomas with byperproliferation of the biliary system in the Fbxw7-deficient liver. We next examined in more detail the long-term effects of Fbxw7 loss in the liver. Macroscopic examination of $M x 1$-Cre $/ F b x w 7^{F / F}$ mice at 50 weeks after $\mathrm{pIpC}$ injection at 8 weeks of age revealed that the mutant liver was enlarged and darker in color compared with the control liver and possessed a rough surface as a result of the presence of several nodules (Figure 5A). We confirmed that the $F b x w 7$ gene was deleted in such nodules (Figure 5B), which were grossly demarcated and readily excised from the liver. Histological examination revealed structural abnormalities characterized by marked dilation of intrahepatic bile ducts as well as apparent proliferation of the biliary system 
A

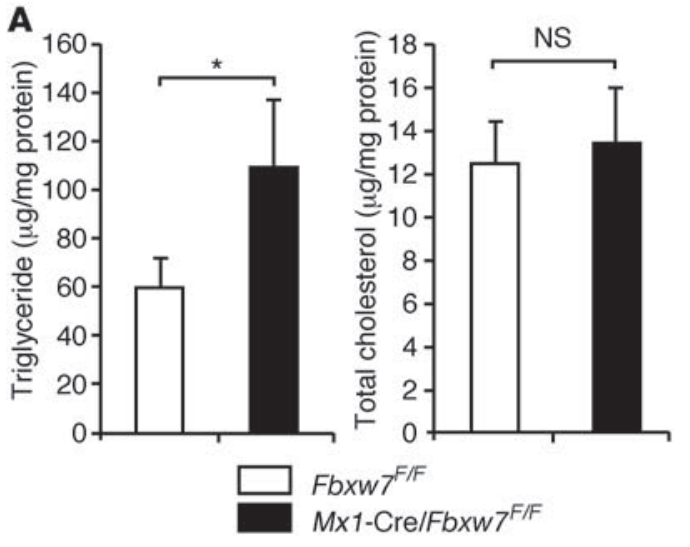

B

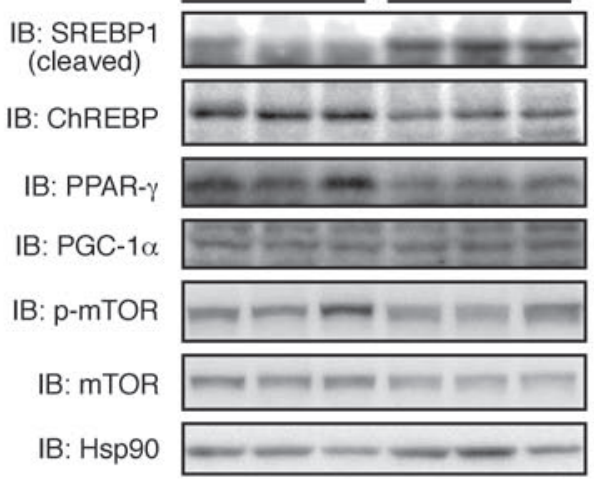

Control Alb-Cre/Fbxw7
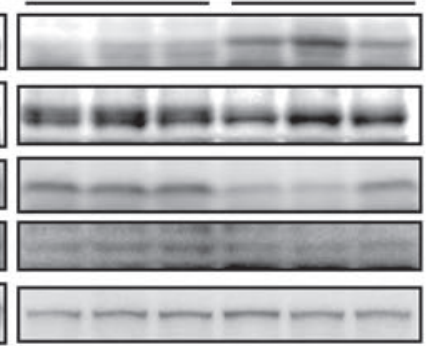
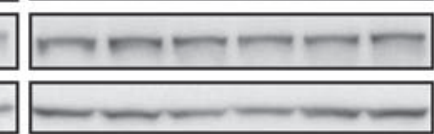

C
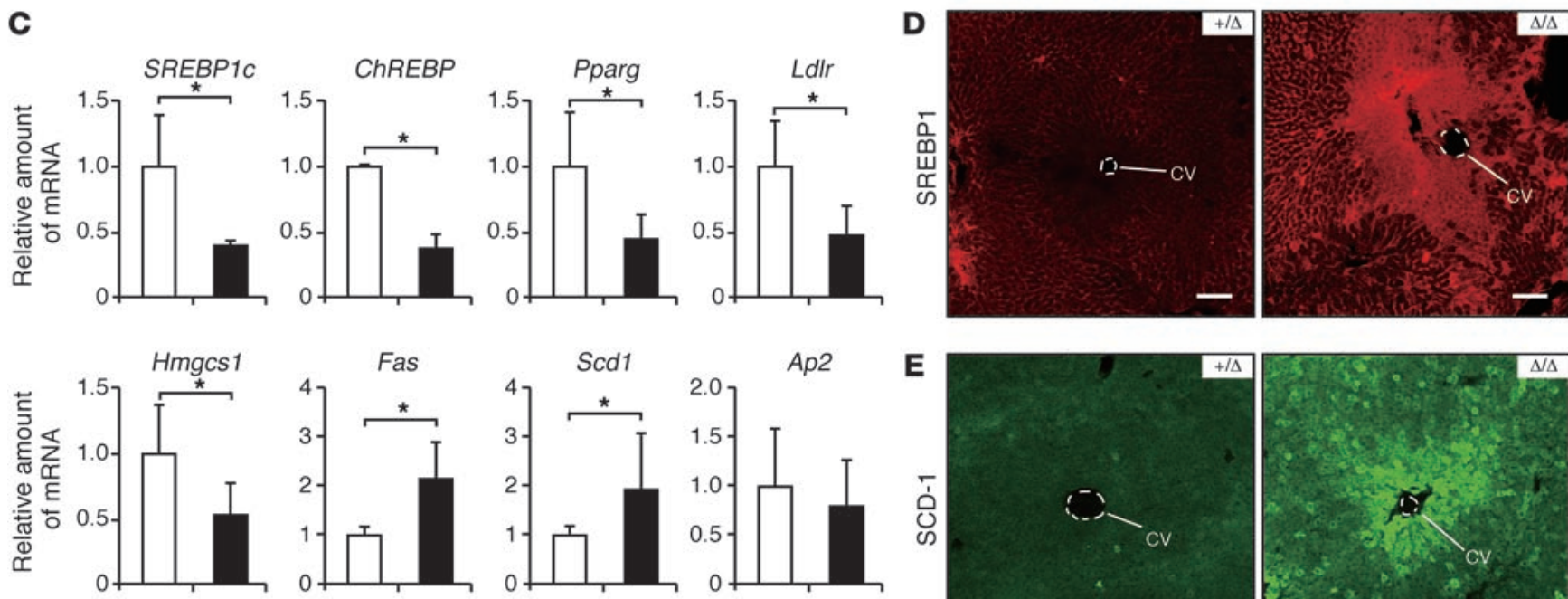

Control

$M \times 1-C r e / F b x w 7^{F / F}$
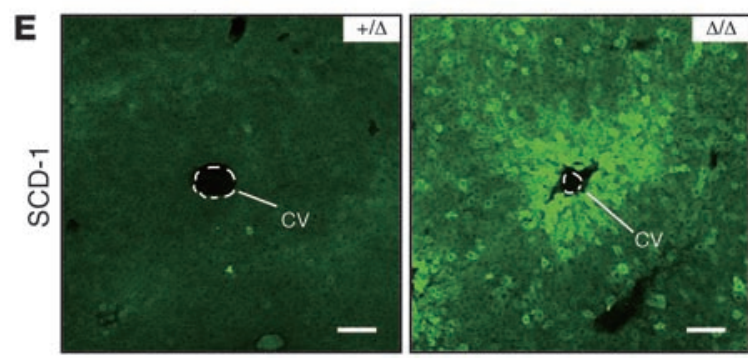

\section{Figure 3}

Deposition of triglyceride and accumulation of SREBP1 in the Fbxw7-deficient liver. (A) Triglyceride and total cholesterol concentrations in the livers of $M \times 1$-Cre/Fbxw $7^{+/ F}$ (control) and $M x 1-C r e / F b x w 7^{F / F}$ mice at 3 weeks after the final injection of plpC, beginning at 8 weeks of age. Data are mean $\pm \mathrm{SD}$ from 3 mice of each genotype. ${ }^{*} P<0.05$. (B) Protein extracts of the livers of $M x 1-\mathrm{Cre} / F b x W 7^{+/ F}$ (control) and $M x 1-\mathrm{Cre} / F b x w 7 F / F$ mice at 3 weeks after the final injection of plpC, beginning at 8 weeks of age, were subjected to IB analysis with antibodies to the indicated proteins (left panel). Liver extracts of $F b x w 7^{F / F}$ (control) and $A / b$-Cre/Fbxw $7^{F / F}$ mice at 12 weeks of age were similarly analyzed (right panel). Three animals were examined for each genotype. Hsp90 was analyzed as a loading control. p-mTOR, phosphorylated mTOR. (C) RT and real-time PCR analysis of the indicated mRNAs in the livers of $M x 1-C r e / F b x w 7^{+/ F}$ (control) and $M x 1-C r e / F b x w 7^{F / F}$ mice treated as in A. Normalized data are expressed relative to the corresponding value for control mice and are mean \pm SD from 3 independent experiments. ${ }^{*} P<0.05$. (D and $\left.\mathbf{E}\right)$ Liver sections of $M \times 1-C r e / F b x w 7^{+/ F}(+/ \Delta)$ and $M \times 1-C r e / F b x w 7^{F / F}(\Delta / \Delta)$ mice, treated as in $\mathbf{A}$, were subjected to immunofluorescence staining with antibodies (D) to SREBP1 and (E) to SCD-1. CV, central vein. Scale bar: $100 \mu \mathrm{m}$.

(Figure 5, C-F); these abnormalities were pathologically diagnosed as hamartomas. Such lesions were also observed, albeit to a lesser extent, in Alb-Cre/Fbxw $7^{F / F}$ mice at as early as 12 weeks of age (Figure $5, \mathrm{G}$ and $\mathrm{H})$. Hamartomas, which are reactive with antibodies to CK19 (Figure 5, I and J), developed in all mutant mice of both genotypes examined $(n=14)$. These results suggested that the loss of Fbxw7 may promote proliferation of the biliary system and shift the development of hepatic stem cells toward the cholangiocyte lineage rather than the hepatocyte lineage.

We examined the abundance of mRNAs for Alb (Figure 5K) and CK19 (Figure 5L) as markers of hepatocyte and cholangiocyte lineages, respectively. The amount of CK19 mRNA in the liver was increased as early as 2 weeks after $\mathrm{PI} C$ injection in $M x 1$-Cre $/ F b x w 7^{F / F}$ mice and showed a more than 40 -fold increase at 50 weeks after Fbxw 7 deletion. In contrast, the abundance of Alb mRNA in the mutant liver at 50 weeks after $\mathrm{pIpC}$ injection was decreased by $40 \%$ compared with that in control liver. These changes in differentiation markers were thus consistent with a marked proliferation of the biliary system in the Fbxw7-deficient liver.

Skewed hepatic differentiation induced by Notch1 accumulation in the Fbxw7-deficient liver. The hepatic cell fate decision is thought to be largely dependent on Notch signaling (39-44). We therefore examined the expression of Notch, a target of Fbxw7, in the Fbxw7deficient liver. Although immunoblot analysis did not reveal an 
A

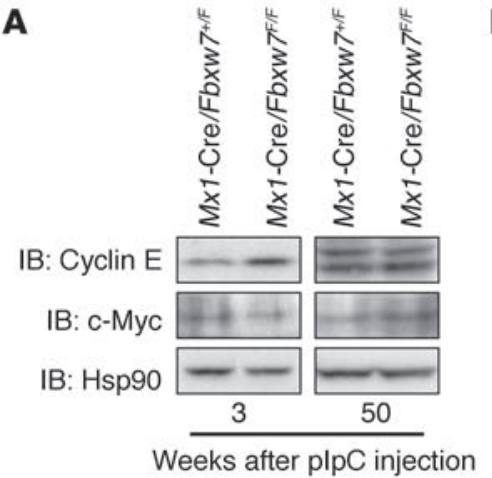

Weeks after plpC injection

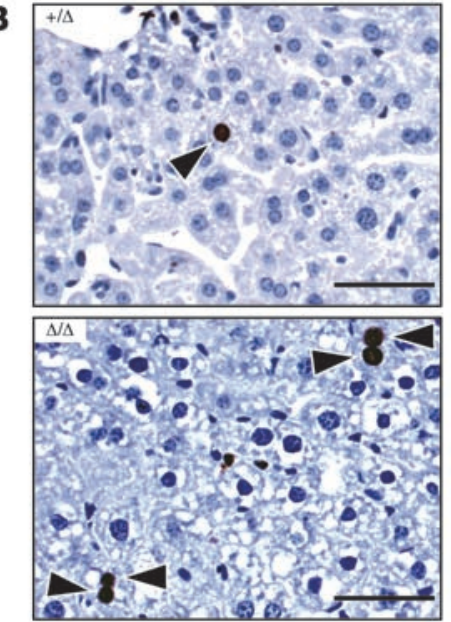

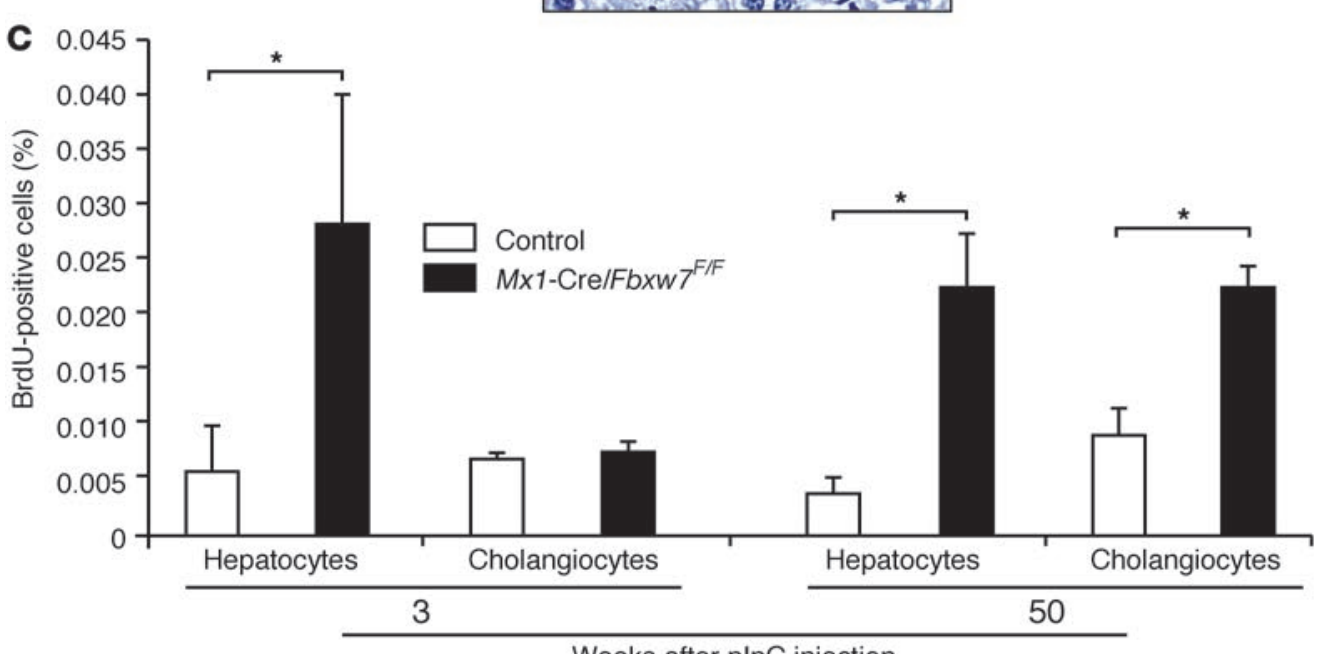

Weeks after plpC injection

D

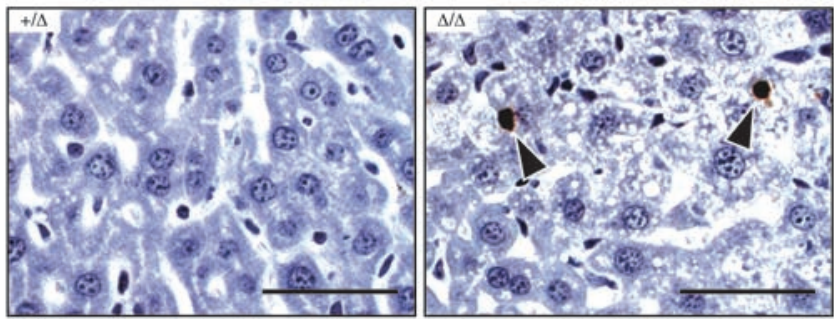

E

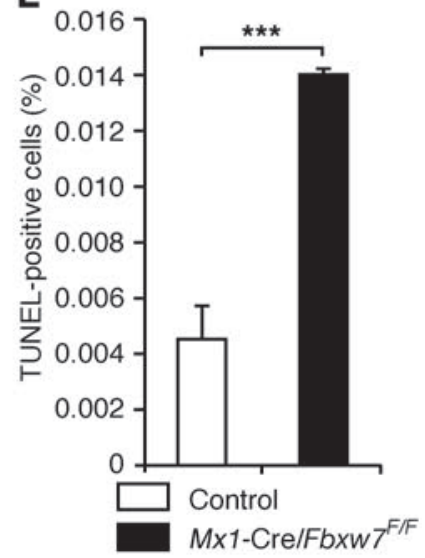

\section{Figure 4}

Increased proliferation and apoptosis of Fbxw7-deficient hepatocytes. (A) IB analysis of cyclin E, c-Myc, and Hsp90 (loading control) in liver extracts from $M \times 1-C r e / F b x w 7^{+/ F}$ and Mx1-Cre/Fbxw7F/F mice at 3 or 50 weeks after $F b x w 7$ deletion by plpC injection, beginning at 8 weeks of age. (B) Representative immunostaining for $\mathrm{BrdU}$ in liver sections from $M \times 1-C r e /$ Fbxw $7^{+/ F}(+/ \Delta)$ and $M \times 1$-Cre/ Fbxw7F/F $(\Delta / \Delta)$ mice injected with BrdU on 3 consecutive days, beginning 3 weeks after the final plpC injection as in A. Arrowheads indicate BrdUpositive nuclei. Scale bar: $50 \mu \mathrm{m}$. (C) The proportion of BrdU-positive hepatocytes or cholangiocytes was determined from immunostaining for $\mathrm{BrdU}$ in combination with that for albumin or CK19 in the livers of $M \times 1$-Cre/Fbxw $7^{+/ F}$ (control) or $M x 1$-Cre/Fbxw7F/F mice at 3 or 50 weeks after deletion of $\mathrm{Fbxw} 7$ as in A. Data are mean \pm SD from 10 fields from 3 mice of each genotype. ${ }^{*} P<0.05$. (D) Representative TUNEL staining for liver sections of $M \times 1-\mathrm{Cre} / \mathrm{Fb} \times w 7^{+/ F}$ $(+/ \Delta)$ and $M \times 1-$ Cre/Fbxw $7^{F / F}$ $(\Delta / \Delta)$ mice 3 weeks after the final plpC injection as in $\mathbf{A}$. Arrowheads indicate TUNELpositive cells. Scale bar: $50 \mu \mathrm{m}$. (E) The proportion of TUNELpositive liver cells was determined from images similar to those in $\mathbf{D}$. Data are mean \pm SD from 3 animals of each genotype. ${ }^{* * *} P<0.005$. increase in the abundance of any of the isoforms of Notch in the liver of $M x 1$-Cre/Fbxw $7^{F / F}$ mice at either 3 or 50 weeks after $F b x w 7$ deletion (data not shown), confocal microscopic analysis revealed that the intracellular domain of Notch1 was highly concentrated in both the cytoplasm and the nucleus of Fbxw7-deficient liver at 3 weeks after $\mathrm{pIpC}$ injection (Figure 6A). Consistent with the observed upregulation of Notch1, the abundance of Notch1 target genes, including those for Hes 1 and Hey1, was simultaneously increased in the livers of $M x 1$-Cre/Fbxw $7^{F / F}$ mice (Figure $6 \mathrm{~B}$ and Supplemental Figure 5A, respectively). At 15 weeks after pIpC injection, Notch1 accumulated in the hepatocyte-like cells residing around the portal area, and these cells were reactive to antibodies to CK7 (Figure 6C), another marker of cholangiocytes, suggesting that such cells might be in the process of transdifferentiation to the cholangiocytes by Notch activation. At 50 weeks after $\mathrm{pIpC}$ injection, Notch 1 was observed in the form of aggregates in the 
A

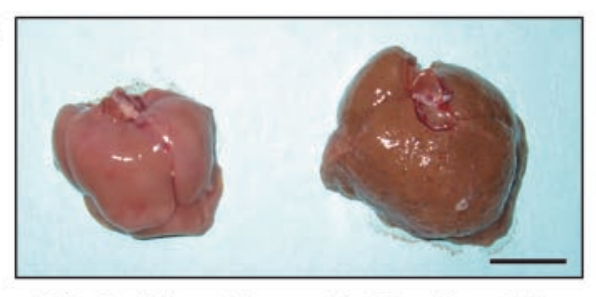

Mx1-Cre/Fbxw $7^{+/ F} \quad M \times 1-C r e / F b x w 7^{F / F}$
B

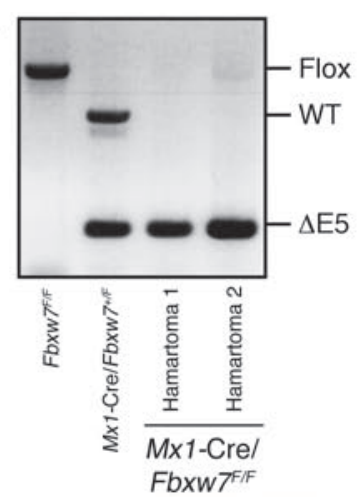

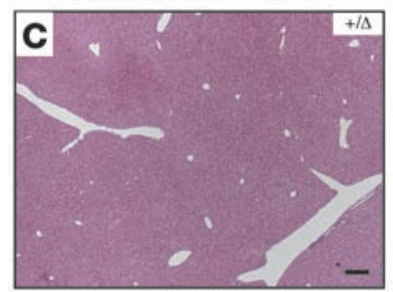
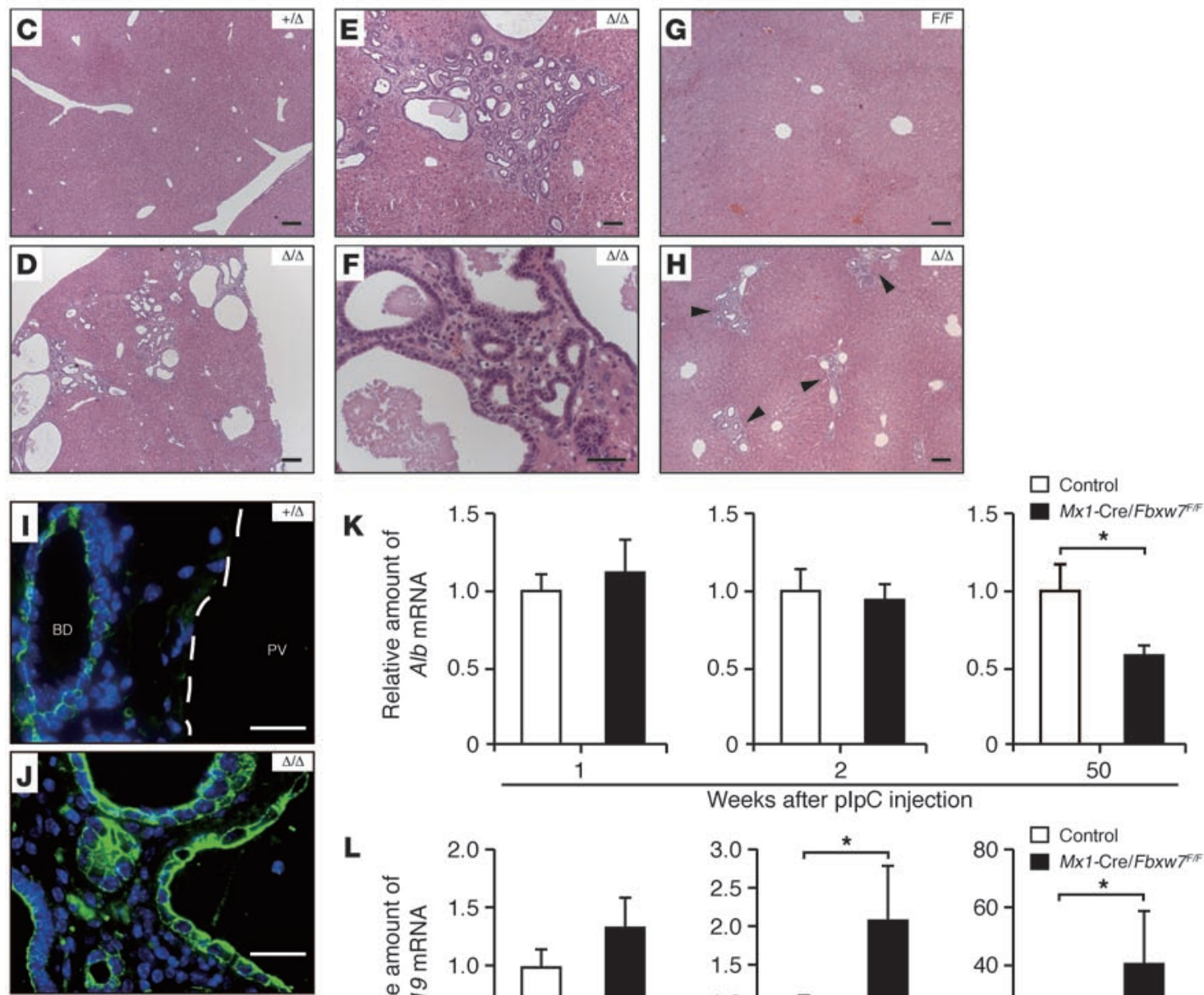

Figure 5

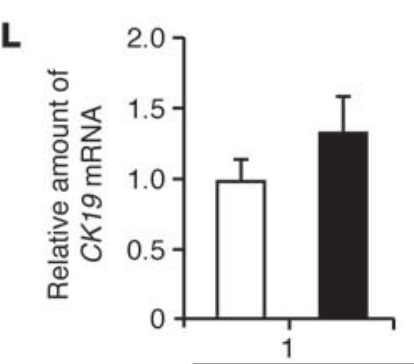

Weeks after plpC injection
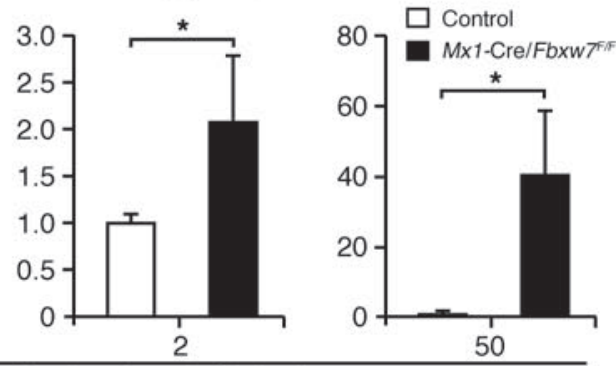

Weeks after plpC injection

Hamartoma development as a result of long-term ablation of Fbxw7 in the liver. (A) Gross appearance of the livers of $M x 1-C r e / F b x w 7^{+/ F}$ and $M \times 1-C r e / F b x w 7^{F / F}$ mice at 50 weeks after the final injection of plpC, beginning at 8 weeks of age. Scale bar: $10 \mathrm{~mm}$. (B) PCR analysis of genomic DNA from the dilated bile ducts excised from hamartomas in the livers of $2 M x 1$-Cre/Fbxw7F/F mice. Genomic DNA from control mice was also analyzed. (C-F) H\&E staining of liver sections from a $M \times 1$-Cre/Fbxw $7^{+/ F}(+/ \Delta)$ mouse $(\mathbf{C})$ and from a $M \times 1-C r e / F b x w 7^{F / F}(\Delta / \Delta)$ mouse $(\mathbf{D}-\mathbf{F})$ that developed hamartoma after Fbxw7 deletion as in A. (G and $\mathbf{H}$ ) H\&E staining of liver sections from $F b x w 7 F / F$ (F/F) and $A / b-C r e / F b x w 7 F / F$ $(\Delta / \Delta)$ mice, respectively, at 12 weeks of age. Arrowheads indicate malformation of the ductal plate. Scale bar: $50 \mu \mathrm{m}(\mathbf{F}) ; 100 \mu \mathrm{m}(\mathbf{E}, \mathbf{G}$, and H); $200 \mu \mathrm{m}$ (C and D). (I and J) Immunofluorescence staining for CK19 in the livers of $M \times 1-C r e / F b x w 7^{+/ F}(+/ \Delta)$ and $M x 1-C r e / F b x w 7 F / F(\Delta / \Delta)$ mice at 50 weeks after the final injection of plpC, beginning at 8 weeks of age. The dashed line indicates the outer boundary of portal vein. PV, portal vein; BD, bile duct. Scale bar: $25 \mu \mathrm{m}$. (K and L) RT and real-time PCR analysis of $A / b$ and $C K 19$ mRNAs, respectively, in the livers of $M \times 1-C r e / F b x w 7^{+/ F}$ (control) and Mx1-Cre/Fbxw F/F mice at 1, 2, or 50 weeks after deletion of $F b x w 7$ as in A. Normalized data are expressed relative to the corresponding value for control mice. Data are mean \pm SD from 3 independent experiments. ${ }^{*} P<0.05$. 
cytoplasm or the nucleus (Supplemental Figure 4A). The increase in Hes 1 or Hey1 was not detected by immunostaining analysis at this period (Supplemental Figure 4B and Supplemental Figure 5B), but the abundance of mRNAs for Hes1, Hey1, and Hey2 was increased in the livers of $M x 1$-Cre $/ F b x w 7^{F / F}$ mice at 50 weeks after pIpC injection (Figure 6D). Neither Notch2, mutations in the gene in which mutations result in Alagille disease, nor Notch3 or Notch4, the expression of both of which is increased in hepatocellular carcinoma, were detected by immunofluorescence analysis in the livers of either control or $M x 1$-Cre/Fbxw $7^{F / F}$ mice (data not shown). However, neither the expression of Notch ligands, such as Dll-1 and Jagged-1, nor that of the Notch cofactor RBP-J in the liver appeared to be affected by the loss of Fbxw7 (Supplemental Figure 6). We also examined the expression of TSC1 and TSC2, given that the loss of function of either TSC1 or TSC2 is known to result in the development of hamartoma in humans. However, no difference in expression of TSC1 or TSC2 was found between Fbxw7-deficient and control mice (Supplemental Figure 6).

To investigate whether the skewed developmental orientation toward the cholangiocyte lineage apparent in the Fbxw7-deficient liver is dependent on Notch 1 accumulation, we examined the differentiation of hepatic stem cells in culture (45). A fraction containing hepatic stem cells was prepared from the livers of $F b x w 7^{+/ F}$ and $F b x w 7^{F / F}$ embryos and was then infected with a retrovirus encoding Cre recombinase or with the empty virus alone to generate $F b x w 7^{+/ F}, F b x w 7^{+/ \Delta}, F b x w 7^{F / F}$, and $F b x w 7^{\Delta / \Delta}$ cells in the presence of HGF and EGF. Immunofluorescence analysis revealed that most of the $F b x w 7^{+/ F}, F b x w 7^{+/ \Delta}$, and $F b x w 7^{F / F}$ cells differentiated into the hepatocyte lineage, characterized by albumin expression, with only a small subset of cells differentiating into the cholangiocyte lineage (Figure 7A). In contrast, the percentage of Fbxw $7^{\Delta / \Delta}$ cells that differentiated into the cholangiocyte lineage, characterized by expression of CK7, was markedly increased compared with that for cells of the control genotypes. To confirm these results in a quantitative manner, we performed RT and real-time PCR analysis of Alb and CK19 mRNAs. Consistent with the immunofluorescence data, the amount of CK19 mRNA was significantly increased in $F b x w 7^{\Delta / \Delta}$ cells compared with that in Fbxw $7^{+/ \Delta}$ cells, whereas the abundance of Alb mRNA did not differ between the 2 genotypes (Figure 7B).

Notch signaling is implicated in the differentiation of liver stem cells into the cholangiocyte lineage. Indeed, immunofluorescence analysis revealed that Notch 1 accumulated in $F b x w 7^{\Delta / \Delta}$ cells to a greater extent than in $F b x w 7^{+/ \Delta}$ cells (Supplemental Figure 7). We therefore examined whether additional ablation of the Notch cofactor RBP-J might correct the abnormal development of Fbxw7-deficient liver stem cells. We generated Fbxw7-deficient hepatic stem cells with additional deletion of either Rbpj or Myc genes and examined the level of CK19 mRNA. The abundance of CK19 mRNA was increased in $F b x w 7^{\Delta / \Delta} M y c^{\Delta / \Delta}$ cells but not in Fbxw $7^{\Delta / \Delta} R b p j^{\Delta / \Delta}$ cells (Figure 7B). These results indicate that the skewed developmental orientation of hepatic stem cells to the cholangiocyte lineage is dependent on Notch 1 accumulation induced by the loss of Fbxw7.

\section{Discussion}

Given that the substrates of Fbxw7 include key proteins that contribute to diverse biological processes, including the cell cycle, cell differentiation, and apoptosis, and that the binding of Fbxw7 to its substrates depends on their phosphorylation, the function of this protein is likely complex. Although much attention has focused on the relation between the accumulation of cyclin $\mathrm{E}$ due to loss of Fbxw7 function and tumorigenesis, Notch degradation by Fbxw7 is critical during embryogenesis, suggesting that Fbxw7 functions in development- and tissue-dependent manners. To provide insight into the physiological and pathological relevance of Fbxw7, we have induced conditional inactivation of Fbxw 7 in several mouse tissues. Our previous studies have shown that ablation of Fbxw 7 in hematopoietic cells or fibroblasts results in abnormalities that are mainly related to the cell cycle and apoptosis. We now show that liver-specific ablation of Fbxw 7 induced fatty liver and abnormal cell differentiation, likely as a result of the accumulation of SREBPs and Notch1, respectively, as well as promoted cell proliferation (Figure 8).

We generated 2 types of mice with liver-specific deficiency of Fbxw7 with the use of the $M x 1$ or Alb gene promoters to drive Cre expression. The phenotypes of Alb-Cre/Fbxw $7^{F / F}$ mice are milder than those induced by acute ablation of Fbxw7 in Mx1$\mathrm{Cre} / \mathrm{Fb} x w 7^{\mathrm{F} / F}$ mice, probably because of the operation of compensatory mechanisms during development in the former animals. In $M x 1$-Cre/Fbxw $7^{F / F}$ mice, it would be expected for $F b x w 7$ to be deleted in cells and tissues other than the liver, such as hematopoietic cells. To exclude the possibility that ablation of Fbxw7 in hematopoietic cell lineages might be responsible for steatohepatitis, we have generated Lck-Cre/Fbxw $7^{F / F}$ and CD4-Cre/Fbxw $7^{F / F}$ mice (in both of which Fbxw7 deletion occurs in T cells), CD19$\mathrm{Cre} / F b x w 7^{F / F}$ mice (Fbxw7 deletion occurs in B cells), and LysM$\mathrm{Cre} / F b x w 7^{F / F}$ mice (Fbxw 7 deletion occurs in myeloid cells). None of these animals showed either fatty liver or hepatic inflammation (data not shown). Furthermore, Alb-Cre/Fbxw $7^{F / F}$ mice manifested pronounced hepatic infiltration of inflammatory cells when they were fed an MCD diet, confirming that the steatohepatitis induced by $F b x w 7$ deletion is attributable to an effect that is intrinsic to the liver.

Nonalcoholic fatty liver disease (NAFLD) is a growing health concern, due to its rapidly increasing prevalence worldwide. NASH is a progressive form of NAFLD that has the potential to develop into hepatocellular carcinoma. We now show that mice with liverspecific ablation of Fbxw7 developed clinicopathologic features similar to those of NAFLD or NASH in humans, including triglyceride deposition around central veins, pericellular fibrosis, infiltration of inflammatory mononuclear cells, and the appearance of Mallory bodies in the liver as well as increases in the serum levels of ALT and AST. However, these animals were not found to develop hepatocellular carcinoma. Genetic mouse models for human NASH have been established by functional deletion of leptin (46) or its receptor (47), phosphatase and tensin homolog (PTEN) (48), NEMO (also known as IKK- $\gamma$ ) (49), interleukin-1 receptor $\alpha(50)$, galectin-3 (51), or retinoic acid receptor $\alpha$ (52). Mice transgenic for SREBP1c also manifest pronounced NASH (53). SREBP1c is degraded in an Fbxw7-dependent manner (16), and we have now shown that it accumulated in the Fbxw7-deficient liver. These findings thus suggest that an Fbxw7-SREBP1 axis plays a key physiological role in the regulation of lipid metabolism in the liver as well as a pathological role in the development of NASH.

Whereas steatosis develops in the acute phase of liver-specific Fbxw7 deficiency, hamartoma develops in the chronic phase. Fbxw7 targets mTOR for degradation (19). The TSC complex, consisting of TSC1 (hamartin) and TSC2 (tuberin), is the major negative regulator of $\mathrm{mTOR}$, and its genetic loss results in $\mathrm{mTOR}$ 
A
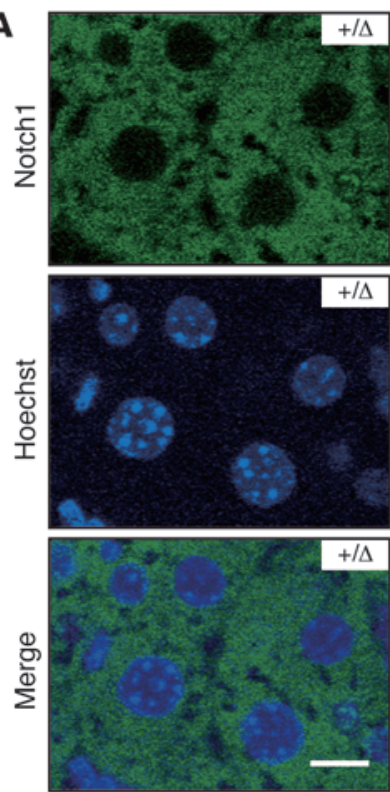

c
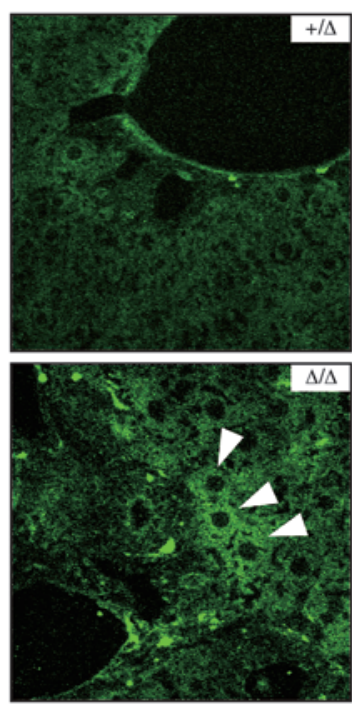

Notch1

D

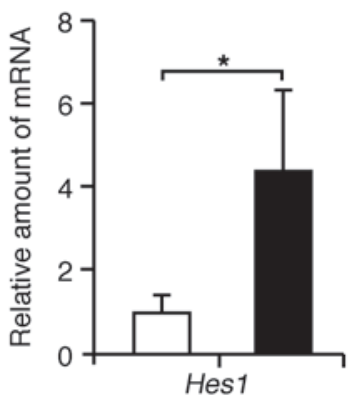

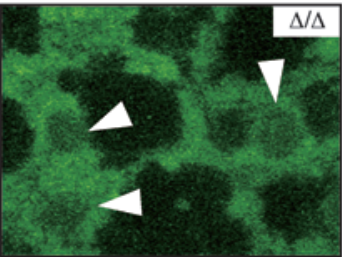
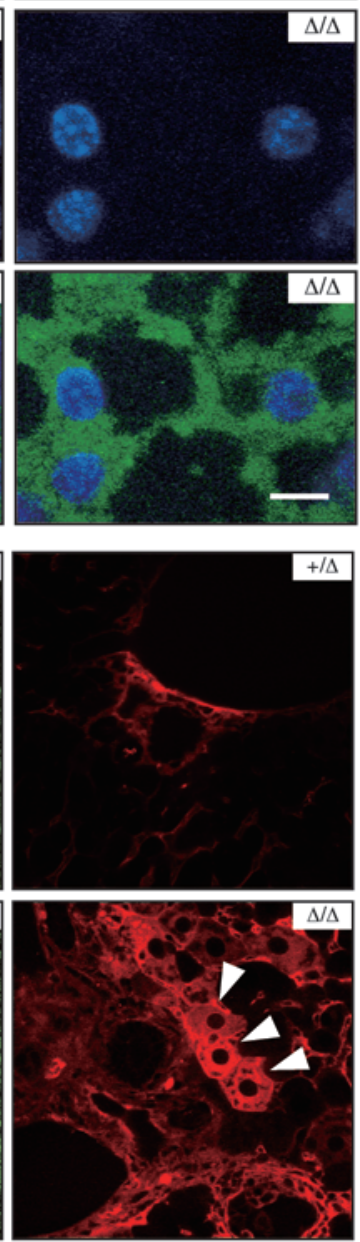

CK7

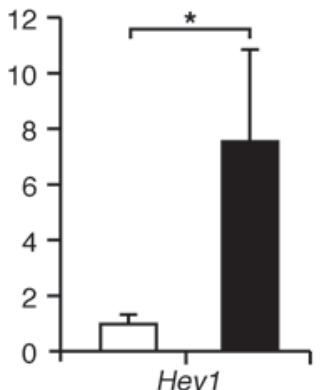

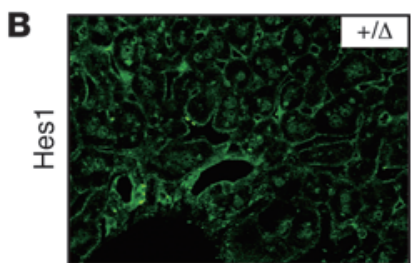
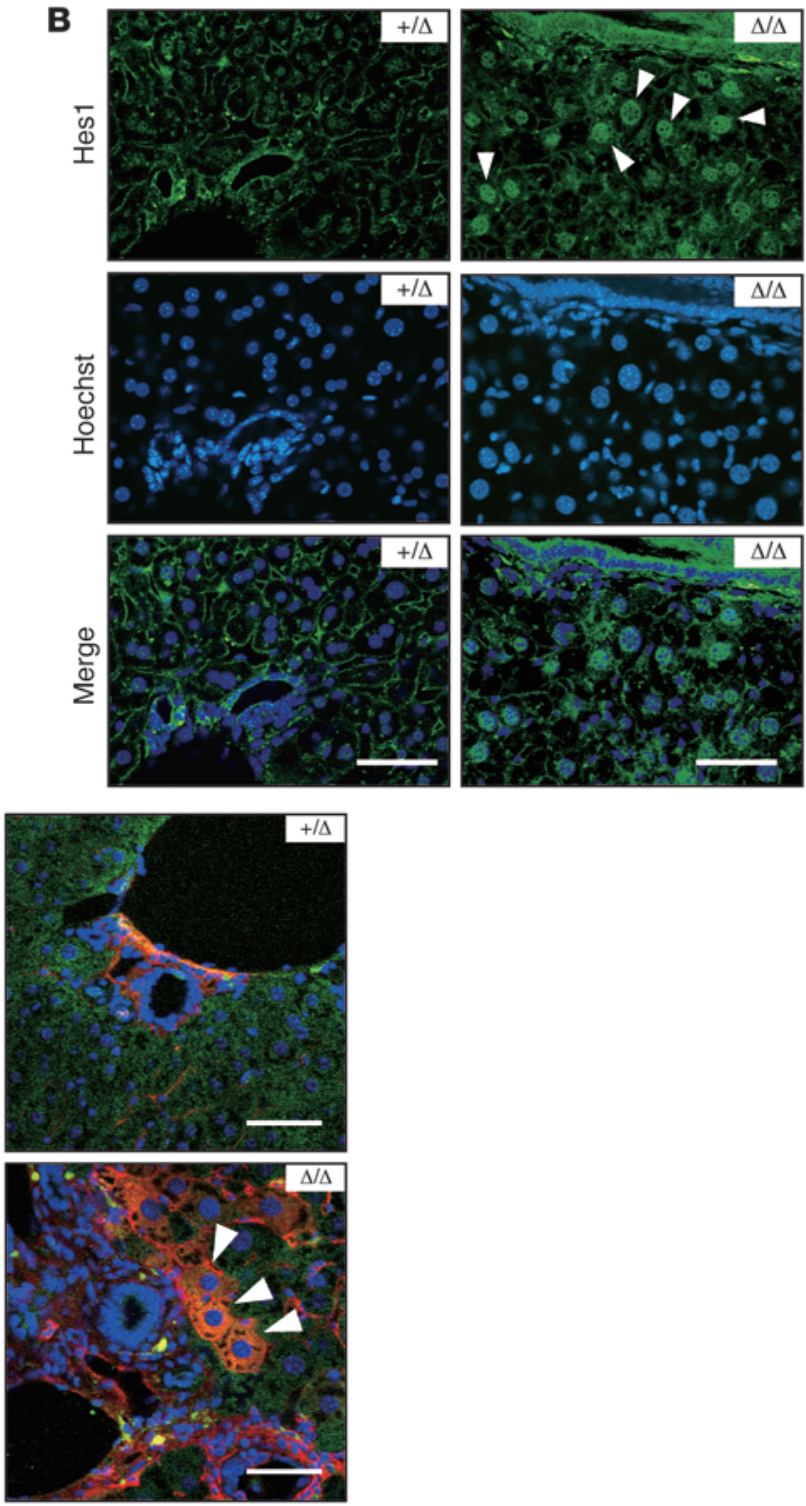

Merge

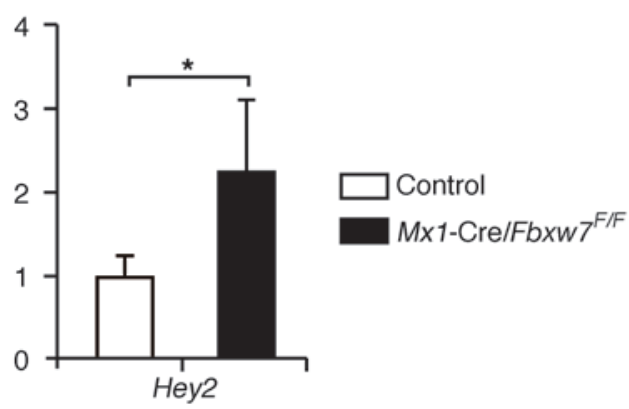

Figure 6

Accumulation of Notch1 and activation of its target genes in the Fbxw7-deficient liver. (A and B) Representative immunostaining for the intracellular domain of $(\mathbf{A})$ Notch1 and for $(\mathbf{B})$ Hes1 in liver sections from $M x 1-C r e / F b x w 7^{+/ F}(+/ \Delta)$ and $M x 1-C r e / F b x w 7 F / F(\Delta / \Delta)$ mice at 3 weeks after $F b x w 7$ deletion by plpC injection, beginning at 8 weeks of age. Arrowheads indicate accumulating (A) Notch1 intracellular domain and (B) Hes1 in the nucleus. (C) Immunofluorescence staining for the intracellular domain of Notch1 and for $C K 7$ in the livers of $M x 1-C r e / F b x w 7^{+/ F}(+/ \Delta)$ and $M x 1-C r e / F b x w 7^{F / F}(\Delta / \Delta)$ mice at 15 weeks after the final injection of plpC, beginning at 8 weeks of age. Intense Notch1 staining was detected in the Fbxw7-deficient liver, and most of the Notch1-positive cells express CK7 (arrowheads). Scale bar: $10 \mu \mathrm{m}$ (A); $50 \mu \mathrm{m}$ (B and C). (D) RT and real-time PCR analysis of Notch target genes in the livers of $M x 1-C r e / F b x w 7^{+/ F}$ (control) and $M x 1-C r e / F b x w 7^{F / F}$ mice at 50 weeks after Fbxw7 deletion. Normalized data for Hes1, Hey1, and Hey2 mRNAs are expressed relative to the corresponding value for control mice and are mean \pm SD from 3 independent experiments. ${ }^{\star} P<0.05$. 
A
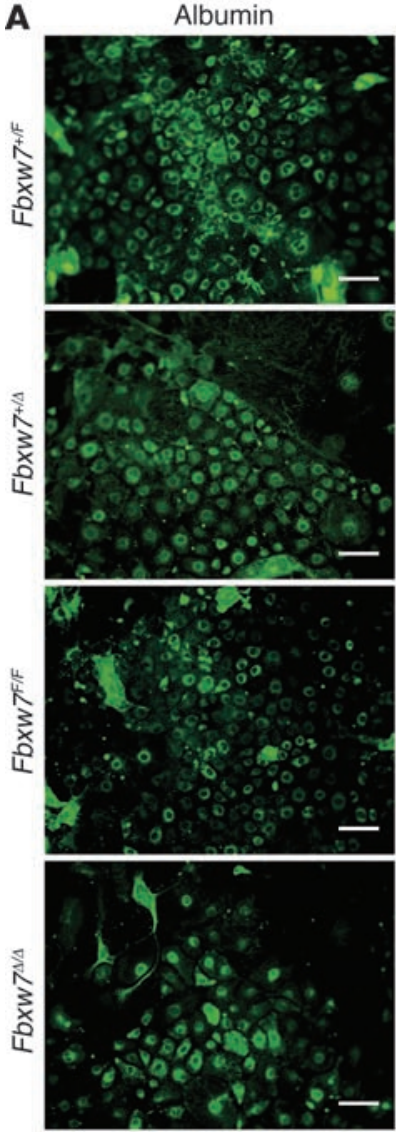

B

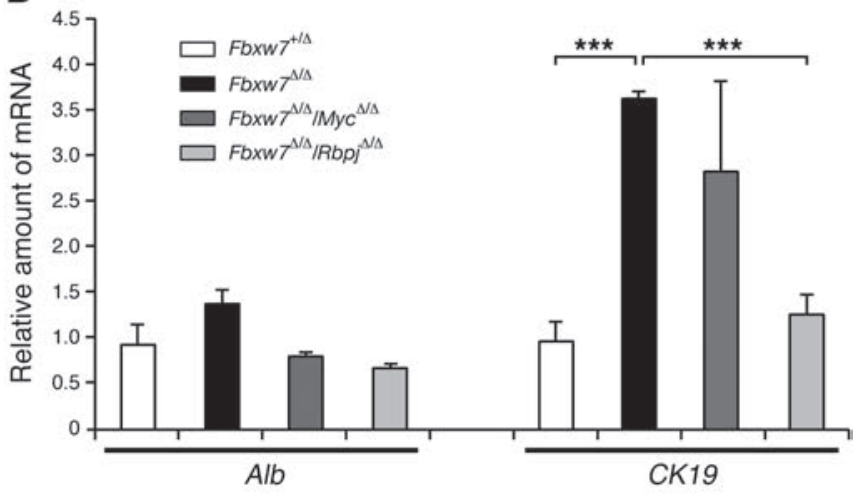

\section{Figure 7}

Skewed differentiation of hepatic stem cells from Fbxw7-deficient mice. (A) Primary cultured liver cells of the indicated genotypes were subjected to immunofluorescence staining with antibodies to albumin or to CK7. Clusters of cells expressing CK7 were apparent only with Fbxw $7^{\Delta / \Delta}$ cells. Scale bar: $50 \mu \mathrm{m}$. (B) RT and real-time PCR analysis of $A / b$ and $C K 19$ mRNAs in primary cultured liver cells of the indicated genotypes. Normalized data for $A / b$ and $C K 19$ mRNAs are expressed relative to the corresponding value for $F b x w 7^{+/ \Delta}$ cells. Data are mean $\pm \mathrm{SD}$ from 3 independent experiments. ${ }^{* \star} P<0.005$. activation and development of hamartoma in humans $(54,55)$. However, the abundance of $\mathrm{mTOR}$ or TSC $1 / 2$ was not altered in the Fbxw7-deficient livers of mice, suggesting that the accumulation of mTOR or the loss of TSC1/2 is not responsible for hamartoma development in these animals. Microscopic examination revealed over proliferation of the biliary system in the hamartomas, suggesting that deregulated differentiation of liver stem cells into the cholangiocyte lineage might be largely responsible for hamartoma development. Liver stem cells are able to differentiate into either the hepatocyte or cholangiocyte lineages, with the Notch signaling pathway having been implicated in regulation of the cell fate decision by skewing differentiation toward the cholangiocyte lineage (41). We have now shown that both Notch1 and its target genes were overexpressed in the Fbxw7-deficient livers of mice and that the abnormal cell differentiation induced by Fbxw7 loss was corrected by the additional loss of the Notch cofactor RBP-J. These results suggest that Notch1 accumulation as a result of Fbxw7 loss is primarily responsible for the abnormal cell differentiation in the Fbxw7-deficient mouse liver. Although the origin of hamartomas as well as the mechanism of their development in the Fbxw7-deficient liver are currently unclear, transient activation of Notch proteins as a result of Fbxw7 loss may lead to a shift in cell differentiation from hepatocytes to cholangiocytes, and the generation of such abnormally differentiated cells might confer a predisposition to hamartoma development that is realized if the cells undergo an additional gene mutation. Mice lacking both Foxa1 and Foxa2 were recently shown to display a similar liver phenotype (hyperplasia of the biliary tree) (56). However, neither differentiation of hepatocytes nor Notch signaling were affected in Foxa1/2-deficient mice, whereas hyperactivation of Notch signaling seems to be attributable to the bile duct hamartoma in Fbxw7-deficient mice. Furthermore, proliferation of relatively small and uniform bile ducts is prominent in Foxa1/2-deficient mice, whereas the abnormal bile ducts in Fbxw7-deficient mice are large and heterogeneous in size. We therefore concluded that the mechanism underlying the development of proliferative bile ducts is likely different between these mutant mice.

Although Fbxw7 had been thought to function primarily in cell cycle control by regulating cyclin E, c-Myc, Notch, and c-Jun, the recent identification of additional substrates has suggested new cell cycle-independent roles for Fbxw7. We now provide genetic evidence that the major substrates of $\mathrm{Fbxw} 7$ in the liver are SREBP1 and Notch1, which accumulate in the Fbxw7-deficient liver and are responsible for liver steatosis and hamartoma development, respectively. These results contrast with our previous observations that deletion of $\mathrm{Fbxw} 7$ in the hematopoietic system and fibroblasts results primarily in deregulation of the cell cycle or of apoptosis due to activation of the p53-dependent checkpoint (34-36). Why does the function of Fbxw7 differ in different tissues? We propose that the biological relevance of Fbxw7 is determined by 3 factors: (a) the expression of Fbxw7; (b) the expression and activation of protein kinases that phosphorylate the Cdc4 phosphodegron, an amino acid sequence that is recognized by Fbxw7; and (c) the expression of substrate molecules. The combination of these 3 factors may define the role of Fbxw7 in a tissue-specific manner, with the different phenotypes associated with Fbxw7 deficiency 


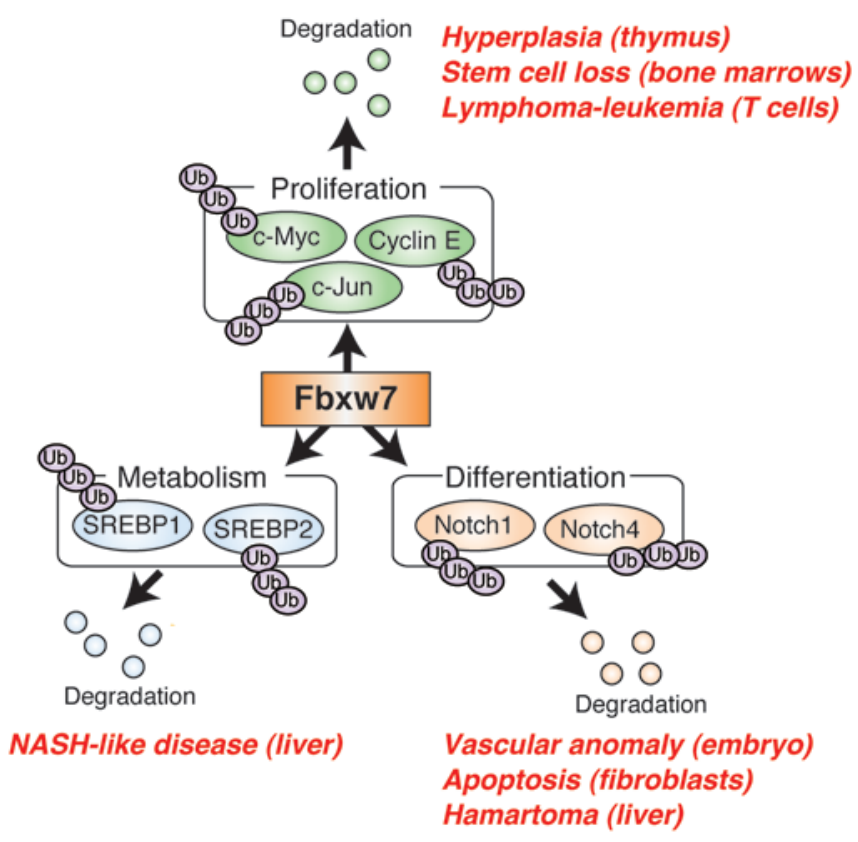

Figure 8

A model for Fbxw7 functions in vivo. Fbxw7 mediates ubiquitin-dependent degradation of substrates in different functional categories. For example, Fbxw7 controls cell proliferation by targeting c-Myc, cyclin E, and c-Jun for degradation. However, it also regulates lipid metabolism and cell differentiation by targeting SREBP and Notch proteins, respectively. Major phenotypes associated with Fbxw7 deficiency in different tissues are shown in red. Ub, ubiquitin.

being attributable to different expression patterns of Fbxw7, its substrates, and kinases that phosphorylate each substrate.

\section{Methods}

Generation of conditional knockout mice. Mice homozygous for the floxed Fbxw 7 allele ( $F b x w 7^{F / F}$ mice) (34) were crossed with $M x 1$-Cre transgenic mice (57) provided by K. Rajewsky (Harvard Medical School, Boston, Massachusetts, USA) or Alb-Cre transgenic mice (58) purchased from The Jackson Laboratory. Expression of Cre recombinase in the resulting offspring of the former cross was induced by i.p. injection of $500 \mu \mathrm{g}$ PIpC (GE Healthcare Biosciences) on 3 alternate days. Deletion of exon 5 of the floxed Fbxw7 allele was confirmed by PCR analysis of genomic DNA as previously described (34). Fbxw $7^{F / F}$ mice were also crossed with $R b p j^{F / F}$ mice (59) provided by T. Honjo (Kyoto University, Kyoto, Japan) or Myc $c^{F / F}$ mice (60) provided by I.M. de Alborán (National Center for Biotechnology, Madrid, Spain). The experimental protocols were approved by the Institutional Animal Care and Use Committee of Kyushu University.

Histological and biochemical analysis. Liver tissue was fixed with $4 \%$ paraformaldehyde in PBS, embedded in paraffin, and stained with $\mathrm{H} \& \mathrm{E}$ or Masson's trichrome solution. Some sections were stained with Oil red $\mathrm{O}$ (Nakalai Tesque) according to standard procedures, in order to examine the extent of lipid accumulation in hepatocytes. Serum levels of AST and ALT were measured with a standard clinical autoanalyzer.

Dietary model of NASH. Mice were fed with an MCD diet (Funabashi Farm) for the indicated periods (see the legend for Figure 2) and analyzed.

Measurement of triglyceride and total cholesterol levels in the liver. Frozen liver tissue was homogenized, and triglyceride and total cholesterol were extracted from the homogenate with chloroform/methanol (2:1, vol/vol), dried, and resuspended in 2-propanol. The amounts of triglyceride and total cholesterol in the extract were measured with the use of Lipidos liquid and Cholescolor liquid kits (Toyobo), respectively.

Immunoblot analysis. Total protein extracts were prepared from liver with RIPA buffer. The extracts $(30 \mu \mathrm{g})$ were subjected to immunoblot analysis as described previously (61) with antibodies to cyclin E (M-20), to c-Myc (N-262), to ChREBP (P-13), or to PPAR- $\gamma$ (E-8), all of which were obtained from Santa Cruz Biotechnology Inc.; with antibodies to Ser ${ }^{2448}$. phosphorylated or total (7C10) forms of mTOR (Cell Signaling Technology); with antibodies to SREBP1 (2A4, NeoMarkers); or with antibodies to PGC-1 $\alpha$ (Chemicon). As a control, each membrane was stripped and then probed with antibodies to Hsp90 (BD Transduction Laboratories).

$R T$ and real-time $P C R$ analysis. Total RNA was extracted from liver using the guanidinium thiocyanate-phenol-chloroform method, purified, and subjected $(1 \mu \mathrm{g})$ to RT with random hexanucleotide primers (ReverTra Ace $\alpha$, Toyobo). The resulting cDNA was subjected to real-time PCR in a reaction mixture that contained $1 \times$ SYBR Green PCR Master Mix (Applied Biosystems) and $200 \mathrm{nM}$ of gene-specific primers. Assays were performed in triplicate with an ABI Prism 7700 Sequence Detector (Applied Biosystems). The PCR protocol comprised 40 cycles of incubation at $60^{\circ} \mathrm{C}$ for 30 seconds and $95^{\circ} \mathrm{C}$ for 5 seconds. The sequences of the PCR primers (sense and antisense, respectively) were $5^{\prime}$ TGCTCCCAGCTGCAGGC- 3 ' and $5^{\prime}$-GCCCGGTAGCTCTGGGTGTA-3' for Fas, 5'-TGGGTTGGCTGCTTGTG-3' and 5'-GCGTGGGCAGGATGAAG-3' for Scd1, $5^{\prime}$-CTGCCGACCTGATGAATTCC-3' and $5^{\prime}$-TAGGGCCATCACACTGTGTC-3' for $L d l r, 5^{\prime}$-GCTCTCCATACAGTGCTACC-3' and $5^{\prime}$-GAGTGAAAGATCATGAAGCC-3' for Hmgcs1, 5'-AGAGATGCCATCTCCAGCCTC-3' and 5'-CTTGGTCTTAGGGTCTTCAGG-3' for ChREBP, $5^{\prime}$-CTGTGAAGTTCAATGCACTGGAA-3' and 5'-CCTCGATGGGCTTCACGTT-3' for Pparg, 5'-CATGGATTGCACATTTGAAG- $3^{\prime}$ and $5^{\prime}$-CCTGTGTCCCCTGTCTCA- 3 ' for SREBP1c, 5'-TCCTGTGCTGCAGCCTTTCTCA-3' and 5'-CCAGGTTCCCACAAAGGCATCA-3' for fatty acid-binding protein 4, 5'-GTCCTACAGATTGACAATGC-3' and 5'-CACGCTCTGGATCTGTGACAG-3' for CK19, 5'-CATGACACCATGCCTGCTGAT-3' and 5'-CTCTGATCTTCAGGAAGTGTAC-3' for Alb, $5^{\prime}$-CATTCCAAGCTAGAGAAGGCAG- $3^{\prime}$ and 5'-TATTTCCCCAACACGCTCG-3' for Hes1, 5'-AAAATGCTGCACACTGCAGG-3' and 5'-CGAGTCCTTCAATGATGCTCAG-3' for Hey1, 5'-AAACGACCTCCGAAAGCGA-3' and 5'-CGGTGAATTGGACCTCATCACT-3' for Hey2, and 5'-GGAACATAGCCGTAAACTGC-3' and 5'-TCACTGTGCCTGAACTTACC- $3^{\prime}$ for $\beta$-tubulin. Reactions for $\beta$-tubulin mRNA were performed concurrently on the same plate as those for the test mRNAs, and results were normalized by the corresponding amount of $\beta$-tubulin mRNA.

BrdU incorporation in vivo. Mice were injected with $\mathrm{BrdU}$ (1 mg, i.p.) on 3 consecutive days. The liver was removed 24 hours after the third injection of BrdU, and BrdU incorporation was examined with an In Situ BrdU Detection Kit (BD Biosciences). BrdU-positive cells were counted in 10 different fields at high $(\times 400)$ magnification, and the percentage of BrdUpositive cells was calculated.

Immunofluorescence microscopy. Liver tissue was fixed with $4 \%$ paraformaldehyde in PBS and sectioned at a thickness of $40 \mu \mathrm{m}$ with a vibratome. Sections were then immunostained with antibodies to the intracellular domain of Notch1 or to SCD-1 (both from Cell Signaling Technology), to Hes1 (AB5702, Millipore), to SREBP1 (2A4, NeoMarkers), to albumin (Biogenesis), to CK19 (45), or to CK7 (MAB3226, Chemicon). Immune complexes were detected with Alexa Fluor 488- or Alexa Fluor 546-conjugated goat antibodies to mouse or rabbit IgG (Invitrogen). Cultured liver cells were also subjected to immunostaining, as described previously (45), with the antibodies to albumin and to CK7. For confocal microscopic analysis, we used Zeiss LSM 510 META Confocal Microscope (Carl Zeiss MicroImaging).

TUNEL assay. The TUNEL assay was performed as described previously (62). In brief, paraffin-embedded sections of liver were treated with $\mathrm{H}_{2} \mathrm{O}_{2}$, 
permeabilized for 15 minutes at $37^{\circ} \mathrm{C}$ with proteinase $\mathrm{K}(20 \mu \mathrm{g} / \mathrm{ml}$, Sigma-Aldrich), and then incubated for 1 hour at $37^{\circ} \mathrm{C}$ with a reaction mixture containing terminal deoxynucleotidyl transferase (Invitrogen) and biotinylated dUTP (Boehringer Ingelheim). Labeled DNA was visualized with an ABC Kit (Vector Laboratories) and diaminobenzidine.

Primary culture offetal hepatocytes. For the preparation of a single-cell suspension, the livers of mice at E13.5 were dissociated in culture medium (DMEM supplemented with $10 \%$ FBS, $\gamma$-insulin [ $1 \mu \mathrm{g} / \mathrm{ml}$, Wako], $0.1 \mu \mathrm{M}$ dexamethasone [Sigma-Aldrich], $10 \mathrm{mM}$ nicotinamide [Sigma-Aldrich], $2 \mathrm{mM}$ L-glutamine [Gibco BRL], $50 \mu \mathrm{M} \beta$-mercaptoethanol [SigmaAldrich], $5 \mathrm{mM}$ HEPES [Wako], and penicillin-streptomycin [Gibco BRL]) by repeated passage of the tissue through the mouth of a pipette. Human recombinant HGF $(50 \mathrm{ng} / \mathrm{ml}$, Sigma-Aldrich) and EGF $(20 \mathrm{ng} / \mathrm{ml}$, SigmaAldrich) were added to the cells at 24 hours after culture initiation. Cells were seeded at a density of $1 \times 10^{6}$ cells per well in 6 -well plates for infection with retroviruses as described below (45).

Gene deletion in cultured cells by retroviral infection. cDNA encoding Cre recombinase was subcloned into the retroviral vector $\mathrm{pMX}$-puro provided by T. Kitamura (University of Tokyo, Tokyo, Japan), and the resulting construct was introduced into Plat E packaging cells (63) with the use of the FuGENE6 reagent (Roche). The resulting culture supernatants containing the recombinant ecotropic retrovirus were harvested and incubated for 24 hours in the presence of Polybrene ( $2 \mu \mathrm{g} / \mathrm{ml}$; Sigma-Aldrich) with proliferating liver cells harboring floxed alleles of $F b x w 7, R b p j$, or $M y c$. The cells were cultured for an additional 24 hours in virus-free med- ium, subjected to selection in medium containing puromycin $(3 \mu \mathrm{g} / \mathrm{ml})$, cultured for 96 hours in puromycin-free medium, and then harvested.

Statistics. Data are presented as mean \pm SD and were analyzed using 2 -tailed Student's $t$ test. A $P$ value of less than 0.05 was considered statistically significant.

\section{Acknowledgments}

We thank T. Honjo for Rbpj floxed mice; I.M. de Alborán for Myc floxed mice; K. Rajewsky for Mx1-Cre transgenic mice; T. Kitamura for pMX-puro; S. Aishima, Y. Nishihara, M. Sakamoto, and R. Irie for discussion; N. Kitajima, Y. Yamada, and K. Takeda for technical assistance; members of our laboratories for comments on the manuscript; and A. Ohta and M. Kimura for help in the preparation of the manuscript. This work was supported in part by a grant from the Ministry of Education, Culture, Sports, Science, and Technology of Japan and by a research grant from the Takeda Science Foundation.

Received for publication August 6, 2009, and accepted in revised form September 29, 2010.

Address correspondence to: Keiichi I. Nakayama, Department of Molecular and Cellular Biology, Medical Institute of Bioregulation, Kyushu University, 3-1-1 Maidashi, Higashi-ku, Fukuoka, Fukuoka 812-8582, Japan. Phone: 81.92.642.6815; Fax: 81.92.642.6819; E-mail: nakayak1@bioreg.kyushu-u.ac.jp.
1. Hershko A, Ciechanover A. The ubiquitin system. Annu Rev Biochem. 1998;67:425-479.

2. Nakayama KI, Nakayama K. Ubiquitin ligases: cell-cycle control and cancer. Nat Rev Cancer. 2006; 6(5):369-381.

3. Frescas D, Pagano M. Deregulated proteolysis by the F-box proteins SKP2 and beta-TrCP: tipping the scales of cancer. Nat Rev Cancer. 2008;8(6):438-449.

4. Welcker M, Clurman BE. FBW7 ubiquitin ligase: a tumour suppressor at the crossroads of cell division, groWTh and differentiation. Nat Rev Cancer. 2008;8(2):83-93.

5. Hubbard EJ, Wu G, Kitajewski J, Greenwald I. sel-10, a negative regulator of lin-12 activity in Caenorhabditis elegans, encodes a member of the CDC4 family of proteins. Genes Dev. 1997;11(23):3182-3193.

6. Sundaram M, Greenwald I. Suppressors of a lin-12 hypomorph define genes that interact with both lin-12 and glp-1 in Caenorbabditis elegans. Genetics. 1993;135(3):765-783.

7. Gupta-Rossi N, et al. Functional interaction between SEL-10, an F-box protein, and the nuclear form of activated Notch 1 receptor. J Biol Chem. 2001;276(37):34371-34378.

8. Oberg C, Li J, Pauley A, Wolf E, Gurney M, Lendahl $\mathrm{U}$. The Notch intracellular domain is ubiquitinated and negatively regulated by the mammalian Sel-10 homolog. J Biol Chem. 2001;276(38):35847-35853.

9. Koepp DM, et al. Phosphorylation-dependent ubiquitination of cyclin $\mathrm{E}$ by the $\mathrm{SCF}^{\mathrm{Fb} w 7}$ ubiquitin ligase. Science. 2001;294(5540):173-177.

10. Moberg KH, Bell DW, Wahrer DC, Haber DA, Hariharan IK. Archipelago regulates Cyclin E levels in Drosophila and is mutated in human cancer cell lines. Nature. 2001;413(6853):311-316.

11. Strohmaier H, Spruck CH, Kaiser P, Won KA, Sangfelt O, Reed SI. Human F-box protein hCdc4 targets cyclin $\mathrm{E}$ for proteolysis and is mutated in a breast cancer cell line. Nature. 2001;413(6853):316-322.

12. Yada M, et al. Phosphorylation-dependent degradation of c-Myc is mediated by the F-box protein Fbw7. EMBO J. 2004;23(10):2116-2125.

13. Welcker M, et al. The Fbw7 tumor suppressor regulates glycogen synthase kinase 3 phosphorylationdependent c-Myc protein degradation. Proc Natl
Acad Sci U S A. 2004;101(24):9085-9090.

14. Nateri AS, Riera-Sans L, Da Costa C, Behrens A. The ubiquitin ligase $\mathrm{SCF}^{\mathrm{Fbw} 7}$ antagonizes apoptotic JNK signaling. Science. 2004;303(5662):1374-1378

15. Wei W, Jin J, Schlisio S, Harper JW, Kaelin WG Jr. The $\mathrm{v}$-Jun point mutation allows c-Jun to escape GSK3dependent recognition and destruction by the Fbw7 ubiquitin ligase. Cancer Cell. 2005;8(1):25-33.

16. Sundqvist A, et al. Control of lipid metabolism by phosphorylation-dependent degradation of the SREBP family of transcription factors by $\mathrm{SCF}^{\mathrm{Fbw}}$. Cell Metab. 2005;1(6):379-391.

17. Punga T, Bengoechea-Alonso MT, Ericsson J Phosphorylation and ubiquitination of the transcription factor sterol regulatory element-binding protein-1 in response to DNA binding. J Biol Chem. 2006;281(35):25278-25286.

18. Bengoechea-Alonso MT, Ericsson J. A phosphorylation cascade controls the degradation of active SREBP1. J Biol Chem. 2009;284(9):5885-5895.

19. Mao JH, et al. FBXW7 targets mTOR for degradation and cooperates with PTEN in tumor suppression. Science. 2008;321(5895):1499-1502.

20. Olson BL, et al. SCFCdc4 acts antagonistically to the PGC-1alpha transcriptional coactivator by targeting it for ubiquitin-mediated proteolysis. Genes Dev. 2008;22(2):252-264.

21. Maser RS, et al. Chromosomally unstable mouse tumours have genomic alterations similar to diverse human cancers. Nature. 2007;447(7147):966-971.

22. Lee JW, et al. Mutational analysis of the hCDC4 gene in gastric carcinomas. Eur J Cancer. 2006; 42(14):2369-2373.

23. Kemp Z, et al. CDC4 mutations occur in a subset of colorectal cancers but are not predicted to cause loss of function and are not associated with chromosomal instability. Cancer Res. 2005; 65(24):11361-11366.

24. Hubalek MM, et al. Cyclin E dysregulation and chromosomal instability in endometrial cancer. Oncogene. 2004;23(23):4187-4192.

25. Koh MS, Ittmann M, Kadmon D, Thompson TC, Leach FS. CDC4 gene expression as potential biomarker for targeted therapy in prostate cancer. Cancer Biol Ther. 2006;5(1):78-83.
26. Calhoun ES, et al. BRAF and FBXW7 (CDC4, FBW7, AGO, SEL10) mutations in distinct subsets of pancreatic cancer: potential therapeutic targets. Am J Pathol. 2003;163(4):1255-1260.

27. Akhoondi S, et al. FBXW7/hCDC4 is a general tumor suppressor in human cancer. Cancer Res. 2007;67(19):9006-9012.

28. Song JH, Schnittke N, Zaat A, Walsh CS, Miller CW. FBXW7 mutation in adult T-cell and B-cell acute lymphocytic leukemias. Lenk Res. 2008; 32(11):1751-1755.

29. Thompson BJ, et al. The $\mathrm{SCF}^{\mathrm{FBW} 7}$ ubiquitin ligase complex as a tumor suppressor in $\mathrm{T}$ cell leukemia. JExp Med. 2007;204(8):1825-1835.

30. O'Neil J, et al. FBW7 mutations in leukemic cells mediate NOTCH pathway activation and resistance to gamma-secretase inhibitors. J Exp Med. 2007;204(8):1813-1824.

31. Malyukova A, et al. The tumor suppressor gene hCDC4 is frequently mutated in human T-cell acute lymphoblastic leukemia with functional consequences for Notch signaling. Cancer Res. 2007;67(12):5611-5616.

32. Tsunematsu R, et al. Mouse Fbw7/Sel-10/Cdc4 is required for notch degradation during vascular development. J Biol Chem. 2004;279(10):9417-9423.

33. Tetzlaff MT, et al. Defective cardiovascular development and elevated cyclin $\mathrm{E}$ and Notch proteins in mice lacking the Fbw7 F-box protein. Proc Natl Acad Sci U S A. 2004;101(10):3338-3345.

34. Onoyama $\mathrm{I}$, et al. Conditional inactivation of Fbxw7 impairs cell-cycle exit during T cell differentiation and results in lymphomatogenesis. J Exp Med. 2007;204(12):2875-2888.

35. Matsuoka S, et al. Fbxw7 acts as a critical failsafe against premature loss of hematopoietic stem cells and development of T-ALL. Genes Dev. 2008;22(8):986-991.

36. Ishikawa Y, Onoyama I, Nakayama KI, Nakayama K. Notch-dependent cell cycle arrest and apoptosis in mouse embryonic fibroblasts lacking Fbxw7. Oncogene. 2008;27(47):6164-6174.

37. Matteoni CA, Younossi ZM, Gramlich T, Boparai N, Liu YC, McCullough AJ. Nonalcoholic fatty liver disease: a spectrum of clinical and pathological 
severity. Gastroenterology. 1999;116(6):1413-1419.

38. Horton JD, et al. Combined analysis of oligonucleotide microarray data from transgenic and knockout mice identifies direct SREBP target genes. Proc Natl Acad Sci U S A. 2003;100(21):12027-12032.

39. Kim BJ, Fulton AB. The genetics and ocular findings of Alagille syndrome. Semin Ophthalmol. 2007;22(4):205-210.

40. Loomes KM, et al. Bile duct proliferation in liverspecific Jag1 conditional knockout mice: effects of gene dosage. Hepatology. 2007;45(2):323-330.

41. Nishikawa Y, et al. Transdifferentiation of mature rat hepatocytes into bile duct-like cells in vitro. $A m$ J Pathol. 2005;166(4):1077-1088.

42. McCright B, Lozier J, Gridley T. A mouse model of Alagille syndrome: Notch2 as a genetic modifier of Jag1 haploinsufficiency. Development. 2002;129(4):1075-1082.

43. McDaniell R, et al. NOTCH2 mutations cause Alagille syndrome, a heterogeneous disorder of the notch signaling pathway. Am J Hum Genet. 2006; 79(1):169-173

44. Kodama Y, Hijikata M, Kageyama R, Shimotohno $\mathrm{K}$, Chiba T. The role of notch signaling in the development of intrahepatic bile ducts. Gastroenterology. 2004;127(6):1775-1786.

45. Suzuki A, et al. Flow-cytometric separation and enrichment of hepatic progenitor cells in the developing mouse liver. Hepatology. 2000;32(6):1230-1239.

46. Leclercq IA, Farrell GC, Schriemer R, Robertson GR. Leptin is essential for the hepatic fibro- genic response to chronic liver injury. J Hepatol. 2002;37(2):206-213.

47. Sahai A, et al. Obese and diabetic $d b / d b$ mice develop marked liver fibrosis in a model of nonalcoholic steatohepatitis: role of short-form leptin receptors and osteopontin. Am J Physiol Gastrointest Liver Physiol. 2004;287(5):G1035-G1043.

48. Horie Y, et al. Hepatocyte-specific Pten deficiency results in steatohepatitis and hepatocellular carcinomas. J Clin Invest. 2004;113(12):1774-1783.

49. Luedde T, et al. Deletion of NEMO/IKKgamma in liver parenchymal cells causes steatohepatitis and hepatocellular carcinoma. Cancer Cell. 2007; 11(2):119-132.

50. Isoda $\mathrm{K}$, et al. Deficiency of interleukin-1 receptor antagonist deteriorates fatty liver and cholesterol metabolism in hypercholesterolemic mice. J Biol Chem. 2005;280(8):7002-7009.

51. Nakanishi Y, et al. Nonalcoholic steatohepatitis and hepatocellular carcinoma in galectin-3 knockout mice. Hepatol Res. 2008;38(12):1241-1251.

52. Yanagitani A, et al. Retinoic acid receptor alpha dominant negative form causes steatohepatitis and liver tumors in transgenic mice. Hepatology. 2004;40(2):366-375.

53. Nakayama H, et al. Transgenic mice expressing nuclear sterol regulatory element-binding protein $1 \mathrm{c}$ in adipose tissue exhibit liver histology similar to nonalcoholic steatohepatitis. Metabolism. 2007;56(4):470-475.

54. Consortium ECTS. Identification and characteriza- tion of the tuberous sclerosis gene on chromosome 16. Cell. 1993;75(7):1305-1315.

55. van Slegtenhorst M, et al. Identification of the tuberous sclerosis gene TSC1 on chromosome 9q34. Science. 1997;277(5327):805-808.

56. Li Z, White P, Tuteja G, Rubins N, Sackett S, Kaestner KH. Foxa1 and Foxa2 regulate bile duct development in mice. J Clin Invest. 2009;119(6):1537-1545.

57. Kuhn R, Schwenk F, Aguet M, Rajewsky K. Inducible gene targeting in mice. Science. 1995; 269(5229):1427-1429.

58. Postic C, Magnuson MA. DNA excision in liver by an albumin-Cre transgene occurs progressively with age. Genesis. 2000;26(2):149-150.

59. Tanigaki K, et al. Regulation of alphabeta/gammadelta $\mathrm{T}$ cell lineage commitment and peripheral $\mathrm{T}$ cell responses by Notch/RBP-J signaling. Immunity. 2004;20(5):611-622.

60. de Alboran IM, et al. Analysis of C-MYC function in normal cells via conditional gene-targeted mutation. Immunity. 2001;14(1):45-55.

61. Kamura T, et al. Cytoplasmic ubiquitin ligase KPC regulates proteolysis of $\mathrm{p} 27^{\mathrm{Kip} 1}$ at $\mathrm{G} 1$ phase. Nat Cell Biol. 2004;6(12):1229-1235.

62. Nishiyama M, Nakayama K, Tsunematsu R, Tsukiyama T, Kikuchi A, Nakayama KI. Early embryonic death in mice lacking the beta-catenin-binding protein Duplin. Mol Cell Biol. 2004;24(19):8386-8394.

63. Morita S, Kojima T, Kitamura T. Plat-E: an efficient and stable system for transient packaging of retroviruses. Gene Ther. 2000;7(12):1063-1066. 